Pacific Journal of Mathematics

SURGERY WITH FINITE FUNDAMENTAL GROUP. II: THE 


\title{
SURGERY WITH FINITE FUNDAMENTAL GROUP II: THE OOZING CONJECTURE
}

\author{
R. JAMES Milgram
}

We determine characteristic class formulae for surgery problems over any compact oriented and closed manifold with finite fundamental group. This essentially evaluates the boundary maps in the surgery exact sequences

$$
\ldots \stackrel{\partial}{\longrightarrow} L_{n+1}^{h}(\mathbf{Z} \pi) \longrightarrow \mathscr{H} \mathscr{T}\left(M^{n}\right) \longrightarrow\left[M^{n}, G / T O P\right] \stackrel{\partial}{\longrightarrow} L_{n}^{h}(\mathbf{Z} \pi) .
$$

(The final step in determining $\partial$ is carried out in a sequel which represents joint work with I. Hambleton, L. Taylor, and B. Williams.) These formulae involve the $L$-genus of $M$, pullbacks of the classes $K_{4 i}$ and $k_{4 i+2}$ in $H^{*}(G / T O P)$ and characteristic classes of the universal covering of $M$ (coming from $\left.H^{*}\left(B_{\pi_{1}(M)}\right)\right)$. It turns out that only classes in the first three of these groups, $*=1,2,3$, are needed. This can be interpreted as saying that only codimension 1,2 , and 3 submanifolds are needed to determine the surgery obstruction. In this form our result was originally conjectured twenty years ago and has become known as the oozing conjecture.

In [5] I showed that there are only four types of surgery obstruction possible for surgery problems over closed manifolds with finite $\pi_{1}$ when the problem is of the form

$$
M \times K^{4 i+2} \longrightarrow M \times S^{4 i+2} .
$$

In order to do this I introduced an intermediate $L$ group $L_{*}^{h}\left(\mathbf{Z}\left(\zeta_{3}\right) \pi\right)$ and maps

$$
e_{\zeta_{3}}: \Omega_{*}\left(B_{\pi}\right) \longrightarrow L_{*}^{h}\left(\mathbf{Z}\left(\zeta_{3}\right) \pi\right)
$$

which factor the product formula above. Then I showed that there were only four types of classes in the image of $e_{\zeta_{3}}$ which could possibly map non-trivially into the surgery groups.

In this paper I obtain characteristic class formulae for the maps $e_{\zeta_{3}}$, at least as regards the specific classes described above. First recall the main results of [5]:

THEOREM A. Let $\pi$ be a finite 2-group, then there are universal kernels $K_{i} \subset L_{i}^{h}\left(\mathbf{Z}\left(\zeta_{3}\right) \pi\right)$ with $L_{0}^{h}\left(\mathbf{Z}\left(\zeta_{3}\right) \pi\right) / K_{0}=\mathbf{Z} / 2$, natural with 
respect to restriction and projection maps, so that, if $e_{\zeta_{3}}(\alpha) \in K_{i}$ then the associated surgery problem above has trivial surgery obstruction. Moreover, the images of $e_{\zeta_{3}}$ in the groups $L_{i}^{h}\left(\mathbf{Z}\left(\zeta_{3}\right) \pi\right) / K_{i}$ are detected by restriction and then projection to the corresponding groups $L_{i}^{h}\left(\mathbf{Z}\left(\zeta_{3}\right) N\right) / K_{i}$ where

(0) the quotient $\mathbf{Z} / 2$ for $i=0$ detects the index of $M$ reduced mod (2),

(1) $N$ runs over $\mathbf{Z} / 2$-quotients of $\pi$ when $i=1$,

(2) $N$ runs over $\mathbf{Z} / 2 \times \mathbf{Z} / 2$ subquotients when $i=2$, and

(3) $N$ runs over quaternion subquotients of $\pi$ when $i=3$,

In this paper we evaluate the map $e_{\zeta_{3}}$ for the basic classes of groups above. For the set of oriented manifolds we obtain complete answers. In the next three theorems we assume $M^{n}$ is oriented.

THEOREM B. When the dimension of $M$ is $4 i+1$, and $\pi_{1}(M)$ is a finite 2-group then the map $e_{\zeta_{3}}$ is non-trivial if and only if it is nontrivial on projecting to some group $\mathbf{Z} / 2$. After projection it is given by the formula

$$
\sigma(\mathrm{id} \times \kappa)=\left\langle V^{2} f^{*}(e),[M]\right\rangle \chi .
$$

Here $\chi$ is the non-zero element in $L_{1}^{h}\left(\mathbf{Z}\left(\zeta_{3}\right) \mathbf{Z} / 2\right) / K_{1}=\mathbf{Z} / 2$ and $V$ is the total $W u$ class of $M$. Finally, $f: M \rightarrow B_{\mathbf{Z} / 2}$ is the classifying map for the universal covering and $e$ is the non-trivial class in $H^{1}\left(B_{\mathbf{Z} / 2} ; \mathbf{Z} / 2\right)$.

In the case $i=2$ the $\mathbf{Z} / 2 \times \mathbf{Z} / 2$ 's usually occur as subgroups of dihedral groups. For this reason we state our next result in terms of dihedral groups.

THEOREM C. When the dimension of $M$ is $4 i+2$ then $e_{\zeta_{3}}$ is detected by restriction, projection to a dihedral group or by projection (factoring through $\pi^{\mathrm{ab}}$ ) onto a group $\mathrm{Z} / 2 \times \mathbf{Z} / 2$. In the case of the dihedral group $L_{2}^{h}\left(\mathbf{Z}\left(\zeta_{3}\right) D\left(2^{i}, 2\right)\right) / K_{2}=(\mathbf{Z} / 2)^{i}$, but there is a unique class $\mu_{i}$ in this quotient which can be in the image of $e_{\zeta_{3}}$. Moreover, there is a unique class

$$
w \in H^{2}\left(B_{D\left(2^{\prime}, 2\right)} ; \mathbf{Z} / 2\right)
$$

which is not in the image of the mod (2) Bochstein and the image of $e_{\zeta_{3}}$ is given by the formula $\left\langle V^{2} f^{*}(w),[M]\right\rangle \mu_{i}$, while in the case $\pi=\mathbf{Z} / 2 \times \mathbf{Z} / 2$ the evaluation is given by the formula

$$
\left\langle V^{2} f^{*}(e \otimes e),[M]\right\rangle \mu_{2} \text {. }
$$


THEOREM D. If the dimension of $M$ is $4 i+3$, then the map is detected by restriction and projection onto quaternion subquotients $\mathscr{Q}_{2^{t}}$ where we have $L_{3}^{h}\left(\mathbf{Z}\left(\zeta_{3}\right) \mathscr{Q}_{2^{\prime}}\right) / K_{3}=\mathbf{Z} / 2$ with generator $\omega_{i}$. The formula for the surgery obstruction is $\left\langle V^{2} f^{*}\left(b_{3}\right),[M]\right\rangle \omega_{i}$, where $b_{3}$ is the non-zero class in $H^{3}\left(B_{Q\left(2^{l}\right)} ; \mathbf{Z} / 2\right)=\mathbf{Z} / 2$.

Note that in each case the deviation from being a pure characteristic class formula is a class $f^{*}(c)$ where dimension $(c) \leq 3$. This is the content of the oozing conjecture for oriented manifolds.

COROllary E. The (codimension 3) oozing conjecture is true for oriented manifolds with finite fundamental group. That is to say, if $\pi$ is a finite group, then the formula for the product with Kervaire obstruction above has the form $\left\langle V^{2} f^{*}\left(\kappa_{*}\right),[M]\right\rangle$ where $\kappa=1+\kappa_{1}+\kappa_{2}+\kappa_{3}$, with the

$$
\kappa_{i} \in H^{i}\left(B_{\pi} ; L_{i+2}^{h}(\mathbf{Z} \pi) \otimes \mathbf{Z} / 2\right)
$$

whenever $M$ is oriented and $\pi_{1}(M)=\pi$.

(From [13], [14] it suffices to verify the result for $\pi$ a finite 2-group, but that is the content of Theorems A-D.)

Of course, Theorems A-D make the determination of the $\kappa$ 's explicit for any finite group $\pi$.

Actually, somewhat more is true. The map $e_{\zeta_{3}}$ is associated to a stable map

$$
s_{\pi}: \Sigma^{0} B_{\pi} \longrightarrow \mathscr{L}_{*}^{h}\left(\mathbf{Z}\left(\zeta_{3}\right) \pi\right)
$$

where $\mathscr{L}\left(\mathbf{Z}\left(\zeta_{3}\right) \pi\right)$ is the Ranicki spectrum [10] for the $L$-groups above. At the prime $2, \mathscr{L}_{*}()$ is a generalized Eilenberg-MacLane space and the oozing conjecture can be regarded as determining the images of the fundamental classes $l \in H^{j}\left(\mathscr{L}_{*}() ; \pi_{j}\left(\mathscr{L}_{*}()\right)\right)$ after restricting to integral homology. But the topological maps have independent interest. Here is what we know in the cases of our model groups.

THEOREM F. The image of $s_{\pi}^{*}: H^{*}\left(\mathscr{L}\left(\mathbf{Z}\left(\zeta_{3}\right) \pi ; \mathbf{Z} / 2\right) \longrightarrow H^{*}\left(B_{\pi} ; \mathbf{Z} / 2\right)\right.$ is always a module over the mod 2 Steenrod algebra, and is generated by $e$ when $\pi=\mathbf{Z} / 2, e \otimes 1,1 \otimes e$, and $e \otimes e$ when $\pi=\mathbf{Z} / 2 \times \mathbf{Z} / 2$. When $\pi=Q\left(2^{i}\right)$ the image is the set of elements of dimension $<4$.

In the case of the dihedral group we have

$$
H^{*}\left(B_{D\left(2^{2}, 2\right)} ; \mathbf{Z} / 2\right)=\mathbf{F}_{2}[x, y, w] /(x y=0),
$$


with $\operatorname{dim}(x)=\operatorname{dim}(y)=1, \operatorname{dim}(w)=2$, and $\operatorname{Sq}^{1}(w)=(x+y) w$ (Theorem 1.25). The generators over the Steenrod algebra of $\operatorname{im}\left(s_{D\left(2^{i}, 2\right)}^{*}\right)$ are then $x, y$, and $w$.

For example, the module generated over $\mathscr{A}(2)$ by $e$ in $H^{*}\left(B_{\mathbf{Z} / 2} ; \mathbf{Z} / 2\right)$ is the $\mathbf{F}_{2}$ vector space with generators $e, e^{2}, e^{4}, \ldots$, $e^{2^{l}}, \ldots$

Our procedure is very close in spirit to that of [3]. There is a commutative diagram

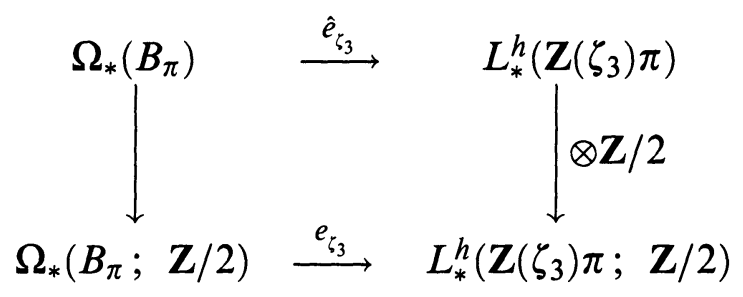

and exactly as was done in [3], characteristic class techniques can be used to obtain from the evaluation of the map $\hat{e}_{\zeta_{3}}$, the image of the associated cohomology map $s_{\pi}^{*}$.

It may be possible to prove an analogue of Theorem $A$ for the groups $L_{*}^{h}\left(\mathbf{Z}\left(\zeta_{3}\right) \pi ; \mathbf{Z} / 2\right)$, but this is not obvious. There are two families of classes (in even dimensions) which we could ignore in Part I, since they were torsion free. But they cannot be ignored here, and moreover, they do not appear to be detected on any reasonably small set of model groups. Nonetheless they should not matter and we make the

Generalized oozing conjecture. For any finite group $\pi$ the image of $\left(s_{\pi}^{*}\right)$ in mod 2 cohomology is generated over $\mathscr{A}(2)$ by a finite number of elements of dimensions $<4$.

In Part 3 of this work [2], using different techniques we obtain more complete information about these mod 2 images, indeed, we obtain sufficient information to prove the conjecture, and this, in turn gives complete characteristic class information for evaluating surgery problems over manifolds with finite fundamental group. This work was completed while the author was a visiting professor at Northwestern University and the University of California at San Diego.

1. Preliminaries. Throughout this paper we assume that all spaces considered have the homotopy types of locally finite $C W$ complexes.

A. Classifying spaces and extensions for finite 2-groups. When $X$ is an associative $H$-space with unit it has a classifying space $B_{X}$ [4], 
which satisfies $\Omega\left(B_{X}\right)$ (the loop space of $B_{X}$ ) is homotopy equivalent to $X$ as a unitary $H$-space. Moreover, $B_{X}$ is unique up to homotopy type. Among the more useful properties of this construction are that $B_{X \times Y}=B_{X} \times B_{Y}$ when $X$ and $Y$ are both associative $H$-spaces with units. Additionally, the construction is natural, so that given an associative $H$-map $f: X \rightarrow Y$ there is induced a map $B_{f}: B_{X} \rightarrow B_{Y}$ with all the expected naturality properties.

When $X$ is a commutative topological group then $B_{X}$ can again be assumed to be a commutative topological group, and in this case the construction can be iterated. (See e.g. [4] for details.)

In particular, if $\pi$ is an Abelian group, then there is an associative, commutative multiplication

$$
B_{\pi} \times B_{\pi} \longrightarrow B_{\pi}
$$

making $B_{\pi}$ into a topological group. (The circle $S^{1} \simeq B_{\mathrm{Z}}$ is a special case of this construction.)

But more is true. Consider a central group extension sequence

$$
H \longrightarrow \pi \longrightarrow \pi / H
$$

where, of course, $H \subset$ center $(\pi)$. It is standard that the isomorphism classes of exact sequences (1.2) with $H, \pi / H$ fixed correspond 1-1 with

$$
\operatorname{Ext}_{\mathbf{Z}(\pi / H)}^{2}(\mathbf{Z} ; A)=H^{2}\left(B_{\pi / H} ; H\right) .
$$

This correspondence has a geometric interpretation.

Note that $B_{\left(B_{H}\right)}=B_{H}^{2}$ is an Eilenberg-MacLane space, and consequently, the set of homotopy classes of maps

$$
\left[X, B_{H}^{2}\right]=H^{2}(X ; H) \text {. }
$$

Also, there is a principal $B_{H}$ fibering

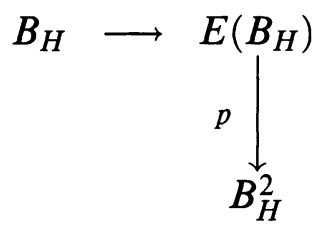

with contractible total space $E\left(B_{H}\right)$, and we have

THEOREM 1.6. The map

$$
B_{p}: B_{\pi} \longrightarrow B_{\pi / H}
$$


induced from (1.2) is a principal fibering with fiber $B_{H}$. Moreover, the classifying map $\gamma_{p}$ for $B_{p}$,

$$
\gamma_{p}: B_{\pi / H} \longrightarrow K(H, 2)
$$

satisfies $\gamma_{p}(l)=\kappa \in H^{2}(\mathbf{Z}(\pi / H) ; H)$ where $\kappa$ is the class in (1.3) corresponding to the extension (1.2).

In particular, corresponding to the multiplication map $H \times \pi \longrightarrow \pi$ (which is itself a homomorphism of groups in this case) there is a map

$$
B_{H} \times B_{\pi} \longrightarrow B_{\pi}
$$

and this map is homotopic to the action of $B_{H}$ on $B_{\pi}$, now regarded as the total space of the principal fibering in 1.6.

EXAMPLES 1.7. The sequence

$$
\mathbf{Z} / 2 \longrightarrow \mathbf{Z} / 4 \longrightarrow \mathbf{Z} / 2
$$

is associated to the non-trivial element $e^{2} \in H^{2}(\mathbf{Z} / 2 ; \mathbf{Z} / 2)=\mathbf{Z} / 2$. The sequence

$$
\mathbf{Z} / 2 \longrightarrow D(4,2) \longrightarrow \mathbf{Z} / 2 \times \mathbf{Z} / 2
$$

is associated to the classes

$$
e_{1} e_{2}, e_{1}\left(e_{1}+e_{2}\right),\left(e_{1}+e_{2}\right) e_{2}
$$

\{all of which are identified via the action of the group of outer automorphisms of $\mathbf{Z} / 2 \times \mathbf{Z} / 2$,

$$
\left(\operatorname{Out}\left((\mathbf{Z} / 2)^{2}\right)=\mathrm{GL}_{2}\left(\mathbf{F}_{2}\right)=\mathscr{S}_{3},\right.
$$

where $\mathscr{S}_{3}$ is the symmetric group on three letters)\}. Similarly, the sequence

$$
\mathbf{Z} / 2 \longrightarrow \mathbf{Z} / 4 \times \mathbf{Z} / 2 \longrightarrow \mathbf{Z} / 2 \times \mathbf{Z} / 2
$$

is associated with the invariants

$$
e_{1}^{2}, e_{2}^{2},\left(e_{1}+e_{2}\right)^{2}=e_{1}^{2}+e_{2}^{2}
$$

and the sequence

$$
\mathbf{Z} / 2 \longrightarrow Q(8) \longrightarrow \mathbf{Z} / 2 \times \mathbf{Z} / 2
$$

is associated to the single element

$$
e_{1}^{2}+e_{1} e_{2}+e_{2}^{2} \text {. }
$$

Here $D\left(2^{i}, 2\right)$ represents the dihedral group of order $2^{i+1}$ with presentation

$$
D\left(2^{i}, 2\right)=\left\{\tau, T \mid \tau^{2}=T^{2^{l}}=(\tau T)^{2}=1\right\}
$$


while $Q\left(2^{i}\right)$ is the quaternion group of order $2^{i}$,

$$
Q\left(2^{i}\right)=\left\{\tau, T \mid \tau^{2}=T^{2^{i-2}}=(\tau T)^{2}\right\} .
$$

B. $\mathbf{Z} / 2$ manifolds and bordism. A $\mathbf{Z} / 2$ manifold is a compact manifold with first Stiefel-Whitney class given as the restriction of an integral cohomology class, together with a specified integral lifting of $w_{1}$. Thus, a $\mathbf{Z} / 2$ manifold consists of a pair

$$
\left\{M, f: M \longrightarrow S^{1}\right\}
$$

where $f$ is defined up to homotopy and $f^{*}(l)$ is the integral lifting of $w_{1}$. If $M^{n}$ and $N^{s}$ are $\mathbf{Z} / 2$-manifolds then

$$
w_{1}\left(M^{n} \times N^{s}\right)=w_{1}\left(M^{n}\right) \otimes 1+1 \otimes w_{1}\left(N^{s}\right)
$$

is again the restriction of a (specific) integral class, so that $M^{n} \times N^{s}$ is also a Z/2-manifold. If the boundary of $M^{n}$ is empty and $M^{n}$ is a $\mathbf{Z} / 2$-manifold then it has a unique non-trivial fundamental class in $H^{n}\left(M^{n} ; \mathbf{Z} / 2\right)=\mathbf{Z} / 2$.

The $\mathbf{Z} / 2$-bordism group of the space $X$, denoted $\Omega_{*}(X ; \mathbf{Z} / 2)$, is the set of bordism classes of maps

$$
f: M^{n} \longrightarrow X
$$

where $M^{n}$ is a $\mathbf{Z} / 2$-manifold without $\partial$. Specifically, $\left(f_{1}, M_{1}^{n}\right) \sim$ $\left(f_{2}, N_{2}^{n}\right)$ if there is a $\mathbf{Z} / 2$-manifold pair $\left(W^{n+1}, p\right)$ with $\partial W=$ $M_{1}^{n} \sqcup N_{2}^{n}$ and $p$ restricted to $M_{i}^{n}$ is $f_{i}$. There is a Hurewicz homomorphism

$$
h: \Omega_{*}(X ; \mathbf{Z} / 2) \longrightarrow H_{*}(X ; \mathbf{Z} / 2)
$$

defined by the rule $h\{(M, f)\}=f_{*}\left(\left[M^{n}\right]\right) \in H_{n}(X ; \mathbf{Z} / 2)$, where $\left[M^{n}\right] \in H_{n}(M ; \mathbf{Z} / 2)$ is the fundamental class.

Likewise, there is an associative pairing

$$
e_{X, Y}: \Omega_{*}(X ; \mathbf{Z} / 2) \otimes \Omega_{*}(Y ; \mathbf{Z} / 2) \longrightarrow \Omega_{*}(X \times Y ; \mathbf{Z} / 2)
$$

defined on generators by

$$
e_{X, Y}\left(\left\{M_{1}, f_{1}\right\} \times\left\{M_{2}, f_{2}\right\}\right)=\left\{M_{1} \times M_{2}, f_{1} \times f_{2}\right\}
$$

so $\Omega_{*}(p t ; \mathbf{Z} / 2)=\mathscr{N}_{*}$ inherits the structure of a graded ring with unit and $\Omega_{*}(X ; \mathbf{Z} / 2)$ is always a graded module over $\mathscr{N} . \mathscr{N}_{0}=\mathbf{Z} / 2$ and there is a natural augmentation $\varepsilon: \mathscr{N} \longrightarrow \mathbf{Z} / 2$ with kernel the ideal $\mathscr{I}=\bigoplus_{j>0} \mathscr{N}_{j}$. Then the composition

$$
h \circ e_{p t, X}: \mathscr{I} \otimes \Omega_{*}(X ; \mathbf{Z} / 2) \longrightarrow H_{*}(X ; \mathbf{Z} / 2)
$$

is the zero map. It follows that there is a well defined homomorphism

$$
\hat{h}: \Omega_{*}(X ; \mathbf{Z} / 2) \otimes_{\mathscr{N}_{*}}\left(\left(\mathscr{N}_{*} / \mathscr{F}\right)=\mathbf{Z} / 2\right) \longrightarrow H_{*}(X ; \mathbf{Z} / 2) .
$$

The following result is well known. 
THEOREM 1.11. (a) $h$ is surjective

(b) $\hat{h}$ above is an isomorphism. In particular

$$
\Omega_{*}(X ; \mathbf{Z} / 2) \cong \mathscr{N}_{*} \otimes_{\mathbf{Z} / 2} H_{*}(X ; \mathbf{Z} / 2)
$$

(c) $\Omega_{*}(X \times Y ; \mathbf{Z} / 2) \cong \Omega_{*}(X ; \mathbf{Z} / 2) \otimes_{\mathscr{N}_{*}} \Omega_{*}(Y ; \mathbf{Z} / 2)$.

As an alternate means of studying $\Omega_{*}(X ; \mathbf{Z} / 2)$, note that we also have the exact sequence

$$
\text { (1.12) } \cdots \longrightarrow \Omega_{n}(X) \stackrel{\times 2}{\longrightarrow} \Omega_{n}(X) \longrightarrow \Omega_{n}(X ; \mathbf{Z} / 2) \stackrel{\partial}{\longrightarrow} \Omega_{n-1}(X) \stackrel{\times 2}{\longrightarrow} \cdots
$$

where $\Omega_{n}(X)$ is the usual oriented bordism group of $X$. In particular, let $\delta M$ be the transverse inverse image of a point on $S^{1}$ under the map $\hat{w}_{1}: M \rightarrow S^{1}$ corresponding to the particular integral lifting of the first Stiefel-Whitney class of $M$, then the class of $(M, f)$ in $\Omega_{n}(X)$ is completely specified by the evaluation of the mod 2 characteristic numbers on $\left(\delta M,\left.f\right|_{\delta M}\right)$, and on the oriented manifold with boundary $(M-\delta M, \partial(M-\delta M), f)$ independently.

The most important group for our applications is $-\mathbf{Z} / 2$ itself, and for this group we now give explicit systems of generators for $\Omega_{*}\left(B_{\mathbf{Z} / 2} ; \mathbf{Z} / 2\right)$ as a $\mathscr{N}_{*}$ module.

Recall that

$$
H_{i}\left(B_{\mathbf{Z} / 2} ; \mathbf{Z} / 2\right)=\mathbf{Z} / 2, \quad i \geq 0,
$$

and, if $\gamma_{i}$ denotes the non-zero element in dimension $i$, then

$$
\gamma_{i} \gamma_{j}=\left(\begin{array}{c}
i+j \\
j
\end{array}\right) \gamma_{i+j}
$$

Thus, as a graded ring,

$$
H_{*}\left(B_{\mathrm{Z} / 2} ; \mathrm{Z} / 2\right) \cong E\left(e_{1}\right) \otimes E\left(e_{2}\right) \otimes E\left(e_{4}\right) \otimes \cdots E\left(e_{2^{i}}\right) \cdots
$$

an exterior algebra on generators in each dimension a power of 2 .

We have an action of $\mathbf{Z} / 2$ on $\mathbf{R P}^{n}$ defined by the rule

$$
T\left(x_{0}, \ldots, x_{n}\right)=\left(x_{0}, \ldots, x_{n-1},-x_{n}\right) .
$$

$T$ is orientation reversing when $n$ is odd. Define

$$
M^{2 n}=S^{1} \times \mathbf{R} \mathbf{P}^{2 n-1} /\{(y, \vec{x}) \sim(-y, T \vec{x})\} .
$$

EXAMPLE 1.15. The first of these, $M^{2}=S^{1} \times_{T} S^{1}$ is just the Klein bottle, with fundamental group $\mathbf{Z} \times_{T} \mathbf{Z}=\left\{T, \tau \mid \tau T \tau^{-1}=T^{-1}\right\}$. The remaining manifolds all have Abelian fundamental group $(\mathbf{Z} / 2) \times \mathbf{Z}$. 


\section{We have}

LEMMA 1.16. $M^{2 n}$ is the projectivized sphere bundle of the vector bundle $\omega=(2 n-1) \varepsilon \oplus \zeta_{1}$ over the circle $S^{1}$, where $\zeta_{1}$ is the nontrivial line bundle and $\varepsilon$ is the trivial line bundle. Consequently

$$
H^{*}\left(M^{2 n} ; \mathbf{Z} / 2\right) \cong H^{*}\left(\mathbf{R P}^{2 n-1} ; \mathbf{Z} / 2\right) \otimes H^{*}\left(S^{1} ; \mathbf{Z} / 2\right)
$$

and, if $e_{1}$ is the generator dual to the section $y \rightarrow(y,(1,0, \cdots, 0))$ (thought of as the generator on the fiber), then

$$
e_{1}^{2 n}=e_{1}^{2 n-1} \cup p^{*}(f)
$$

is the non-trivial class.

(Recall that $e_{1}^{2 n}=\sum w_{i}(\omega) e^{2 n-i}$ \{see e.g. [8]\} where the $w_{i}(\omega)$ are the Stiefel-Whitney classes of the bundle $\omega$, and, since $W(\omega)=$ $1+p^{*}(f)$, the result follows.)

COROllaRY 1.17. (a) $M^{2 n}$ is bordant to zero in $\Omega_{2 n}(p t ; Z / 2)$.

(b) The ith Wu class of $M^{2 n}$ is given as

$$
V_{i}=\left(\begin{array}{c}
2 n-i-1 \\
i
\end{array}\right) e_{1}^{i}+\left(\begin{array}{c}
2 n-i-1 \\
i-1
\end{array}\right) e_{1}^{i-1} p^{*}(f) .
$$

(Indeed,

$$
\begin{gathered}
\operatorname{Sq}^{i}\left(e_{1}^{2 n-i}\right)=\left(\begin{array}{c}
2 n-i \\
i
\end{array}\right) e_{1}^{2 n} \\
\operatorname{Sq}^{i}\left(e_{1}^{2 n-i-1} p^{*}(f)\right)=\left(\begin{array}{c}
2 n-i-1 \\
i
\end{array}\right) e_{1}^{2 n}
\end{gathered}
$$

and it is easily checked that $V_{i}$ above when cupped with these classes gives the same result.)

REMARK 1.18. For future use, let $M^{1}=S^{1}$, and $M(i)=M^{2^{i}}$. Then for each of the spaces $M(i), i=0,1,2, \ldots$ we have that $V^{2}$ is just the class 1 in dimension 0 , while $V \mathrm{Sq}^{1}(V),\left(\mathrm{Sq}^{1} V\right)^{2}$ are identically zero.

Corollary 1.19. Let $p_{m}: M^{2 n} \rightarrow B_{\mathbf{Z} / 2}$ be the map sending the fundamental class in $H^{1}\left(B_{\mathbf{Z} / 2} ; \mathbf{Z} / 2\right)$ to $e_{1}$, then the composite map

$$
M^{2 n} \times M^{2 n} \stackrel{p_{n} \times p_{n}}{\longrightarrow} B_{\mathbf{Z} / 2} \times B_{\mathbf{Z} / 2} \stackrel{e}{\longrightarrow} B_{\mathbf{Z} / 2}
$$

represents 0 in $\Omega_{4 n}\left(B_{\mathbf{Z} / 2} ; \mathbf{Z} / 2\right)$. 
Proof.

$$
\delta\left(M^{2 n} \times M^{2 n}\right)=\left(\mathbf{R P}^{2 n-1} \times \mathbf{R} \mathbf{P}^{2 n-1}\right) \times_{T} S^{1}
$$

where $T$ acts diagonally on $\mathbf{R} \mathbf{P}^{2 n-1} \times \mathbf{R} \mathbf{P}^{2 n-1}$, and freely on $S^{1}$.

The interchange map on $M^{2 n} \times M^{2 n}$ restricts to the map on $\delta\left(M^{2 n} \times M^{2 n}\right)$ which just interchanges the two copies of $\mathbf{R P}^{2 n-1}$ and is fixed on $S^{1}$. In both $\delta\left(M^{2 n} \times M^{2 n}\right)$ and $M^{2 n} \times M^{2 n}$ the class $\left(e(p \times p)^{*}\right) l=(\tau+1) A$, where $\tau$ is the cohomology map induced by the involution above. From this it follows (since the Stiefel-Whitney classes of $M^{2 n} \times M^{2 n}$ and $\delta\left(M^{2 n} \times M^{2 n}\right)$ are invariant under $\left.\tau\right)$ that every characteristic class in top dimension is of the form $(\tau+1) B$ for some $B$, but since $\tau=1$ in dimension $2 n$ all these classes are zero and the result follows.

COROLlaRY 1.20. $\Omega_{*}\left(B_{(\mathbf{Z} / 2)^{n}} ; \mathbf{Z} / 2\right)=\bigotimes_{j=1 \cdots n} \otimes_{i} E\left(\left\{M(i), f_{j}\right\}\right)$, where $f_{j}$ maps $M(i)$ into the jth summand of $B_{(\mathbf{Z} / 2)^{n}}=\left(B_{\mathbf{Z} / 2}\right)^{n}$ as a ring over $\mathscr{N}_{*}$.

C. The homology and bordism of the dihedral groups $D\left(2^{n}, 2\right)$ and the quaternion groups $Q\left(2^{i+2}\right)$. We have the following central extension sequences for $D\left(2^{i}, 2\right)$, and $Q\left(2^{i}\right)$,

$$
\begin{aligned}
& 1 \longrightarrow \mathbf{Z} / 2 \longrightarrow D\left(2^{i}, 2\right) \longrightarrow D\left(2^{i-1}, 2\right) \longrightarrow 1 \\
& 1 \longrightarrow \mathbf{Z} / 2 \longrightarrow Q\left(2^{i+2}\right) \longrightarrow D\left(2^{i}, 2\right) \longrightarrow 1 .
\end{aligned}
$$

Thus, we proceed by using the first of these sequences to study $D\left(2^{i}, 2\right)$, and then use the second to obtain $Q\left(2^{i}\right)$ from our knowledge of the dihedral groups.

A more symmetric presentation (and one more adapted to the use of 1.21 than that in 1.8 ) is given by

$$
D\left(2^{i}, 2\right)=\left\{x, y \mid x^{2}=y^{2}=(x y)^{2^{l}}=1\right\}
$$

(set $y=\tau T, x=\tau$ in (1.8)), and we have two homomorphisms

$$
\begin{gathered}
\phi_{1}^{i}, \phi_{2}^{i}: \mathbf{Z} / 2 \times Z / 2 \longrightarrow D\left(2^{i}, 2\right), \\
\phi_{j}^{i}(\tau)=(x y)^{2^{1-1}}, \quad \phi_{1}^{i}(T)=x, \quad \phi_{2}^{i}(T)=y .
\end{gathered}
$$

Note also that $\left(D\left(2^{i}, 2\right)\right)^{\mathrm{ab}}=\mathbf{Z} / 2 \times \mathbf{Z} / 2$, and let the projection be 
denoted by $p$. We then have the commutative diagram
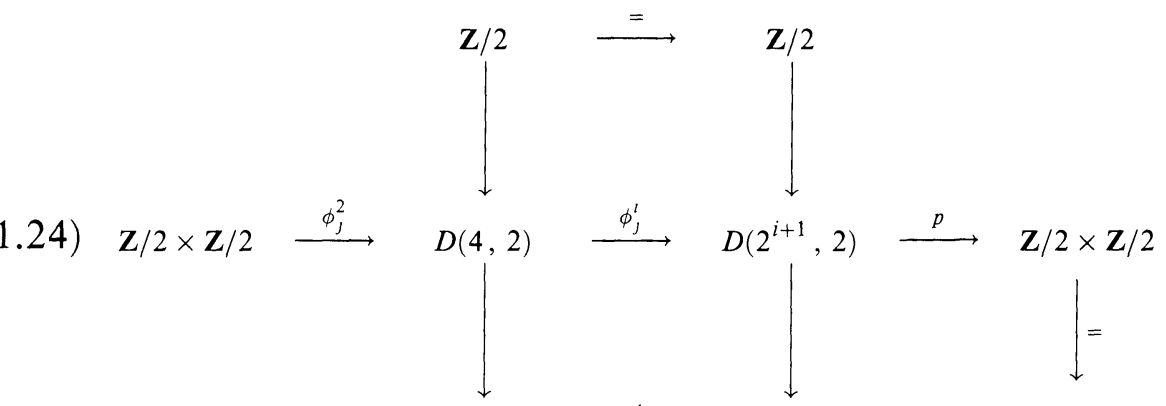

$$
\mathbf{Z} / 2 \times \mathbf{Z} / 2 \stackrel{\phi_{j}^{\prime}}{\longrightarrow} D\left(2^{l}, 2\right) \stackrel{p}{\longrightarrow} \mathbf{Z} / 2 \times \mathbf{Z} / 2
$$

and the composite $\phi_{j}^{i} \phi_{j}^{2}$ is $\phi_{j}^{i+1}$. Specific classes $\langle x\rangle,\langle y\rangle$ in $H^{1}\left(D\left(2^{i}, 2\right) ; \mathbf{Z} / 2\right)$ are given as the composites $p^{*} \lambda_{j}^{*}(e)$ where

$$
\lambda_{j}: \mathbf{Z} / 2 \times \mathbf{Z} / 2 \longrightarrow \mathbf{Z} / 2
$$

are the projections onto the $j$ th factors (so e.g. $\lambda_{1} p(x)=T, \lambda_{1} p(y)=$ $1)$ and $e$ is the non-trivial class in $H^{1}(\mathbf{Z} / 2 ; \mathbf{Z} / 2)$. Then we have

THEOREM 1.25. (a) $H^{*}\left(D\left(2^{i}, 2\right) ; \mathbf{Z} / 2\right)=\mathbf{F}_{2}[x, y, w] / x y=0$, with $x, y$ both 1-dimensional, and $w$ 2-dimensional. Moreover, $S q^{1}(w)=(x+y) w$, and the $k$-invariant for the extension sequence above (with $i$ replaced by $i+1$ ) is $w$.

(b) The two inclusions

$$
\phi_{j}^{i}: \mathbf{Z} / 2 \times \mathbf{Z} / 2 \longrightarrow D\left(2^{i}, 2\right),
$$

together surject in mod 2 homology.

(c) The inclusion of the center $\alpha: \mathbf{Z} / 2 \longrightarrow D\left(2^{i}, 2\right)$ induces

$$
\alpha^{*}: H^{*}\left(D\left(2^{i}, 2\right) ; \mathbf{Z} / 2\right) \longrightarrow H^{*}(\mathbf{Z} / 2 ; \mathbf{Z} / 2),
$$

and $\alpha^{*}(w)=e^{2}, \alpha^{*}(x)=\alpha^{*}(y)=0$.

(d) The two maps $\phi_{j}^{i}$ satisfy $\phi_{j}^{i *}(w)=y^{2}+x y$, while $\phi_{1}^{i *}(x)=x$, $\phi_{1}^{i *}(y)=0, \phi_{2}^{i *}(x)=0, \phi_{2}^{i *}(y)=x$.

Proof. The proof is by induction. First some general remarks are required. Assuming that the result is true for $D\left(2^{i}, 2\right)$ we note that the $k$-invariant for $D\left(2^{i+1}, 2\right)$ must be $w_{i}$. Indeed, from (1.24) we have that $\phi_{j}^{i}(\kappa)$ is a suitable $k$-invariant for $D(4,2)$. But, by the induction, the only image which is suitable for such a $k$-invariant is $y^{2}+x y$. Moreover, there must be some element $\gamma$ in $H^{2}\left(D\left(2^{i+1}, 2\right) ; \mathbf{Z} / 2\right)$ with composite image under all four of the maps $\phi_{j}^{2} \phi_{k}^{i}$ the $k$-invariant for a dihedral extension. 
But, as we just observed, there is only one such image from $H^{2}(D(4,2) ; \mathbf{Z} / 2)$. So there is an element in $H^{2}\left(D\left(2^{i+1}, 2\right) ; \mathbf{Z} / 2\right)$ which we can take to be our new class $w_{i+1}$. Moreover, again referring to (1.24) and the definitions of $x, y$, it is direct to see that the maps $\phi_{j}^{i+1}$ behave correctly on $x$ and $y$. So, to continue the induction we need only show the injectivity of these two maps in cohomology, and the fact that $\operatorname{Sq}^{1}\left(w_{i+1}\right)=(x+y) w_{i+1}$.

For the moment let us assume the injectivity. Then

$$
\operatorname{Sq}^{1}\left(\phi_{j}^{i+1}\left(w_{i+1}\right)\right)=x y^{2}+x^{2} y=x\left(\phi_{j}^{i+1}\left(w_{i+1}\right)\right) .
$$

But the only class which can have this image under both maps is $(x+y) w_{i+1}$, and the proof will be complete once we have demonstrated that $H^{*}\left(D\left(2^{i+1}, 2\right) ; \mathbf{Z} / 2\right)$ has the form stated in the theorem.

For this consider the Serre spectral sequence of the fibering associated to the central extension giving $D\left(2^{i+1}, 2\right)$. Its $E_{2}^{i, j}$ term is $H^{i}\left(D\left(2^{i}, 2\right) ; H^{j}(\mathbf{Z} / 2 ; \mathbf{Z} / 2)\right)$ which can be written as

$$
E_{2}^{i, j}=H^{i}\left(D\left(2^{i}, 2\right) ; \mathbf{Z} / 2\right) \otimes H^{j}(\mathbf{Z} / 2 ; \mathbf{Z} / 2) .
$$

The differential is completely determined by $d^{2}\left(e_{1}\right)=w_{i}$, and we have that

$$
E_{3}^{*, *}=\{\mathbf{Z} / 2[x, y] /(x y=0)\} \otimes \mathbf{Z} / 2\left[e^{2}\right] .
$$

But $d^{3}\left(e^{2}\right)=\operatorname{Sq}^{1}\left(w_{i}\right)=(x+y) w_{i}=0$ in $E_{3}^{3,0}$, so $E_{3}=E_{\infty}$, and this completes the inductive step.

The proof of 1.25 will be completed once we show we can start the induction. So construct $D(4,2)$ from the extension sequence

$$
\mathbf{Z} / 2 \longrightarrow D(4,2) \longrightarrow \mathbf{Z} / 2 \times \mathbf{Z} / 2
$$

with $k$-invariant $x y$. First, a spectral sequence argument identical to that above shows $H^{*}(D(4,2) ; \mathbf{Z} / 2)=\mathbf{Z} / 2[x, y, w] /(x y=0)$. Next, we must check that the image of $w_{2}$ under the two maps $\phi_{j}^{2}$ is as stated. Note first that on going up to $D(8,2)$ the original center of $D(4,2)$ is embedded into the extension sequence

$$
\mathrm{Z} / 2 \longrightarrow \mathrm{Z} / 4 \longrightarrow \mathrm{Z} / 2
$$

This implies that $y^{2}$ must be part of the image of $w$. On the other hand, the image of $w$ must also include $x y$, since the extension induced on $\mathbf{Z} / 2 \times \mathbf{Z} / 2$ is $D(4,2)$. Now the proof is complete.

Thus, the discussion above for $B_{\mathbf{Z} / 2 \times \mathbf{Z} / 2}$ gives us control of the $\mathbf{Z} / 2$-bordism of $B_{D\left(2^{i}, 2\right)}$. 
COROLLARY 1.26.

$$
H^{*}\left(Q\left(2^{i}\right) ; \mathbf{Z} / 2\right)=\mathbf{Z} / 2\left[x, y, v_{i}\right] /\left(x^{3}=y^{3}=x y=0\right)
$$

for $i>3$, while

$$
\begin{aligned}
& H^{*}(Q(8) ; \mathbf{Z} / 2) \\
& \quad=\mathbf{Z} / 2\left[x, y, v_{1}\right] /\left(x^{2}+x y+y^{2}=0, x y^{2}+x^{2} y=0\right)
\end{aligned}
$$

where $v_{i}$ has dimension 4.

Proof. Consider the diagram of central extensions

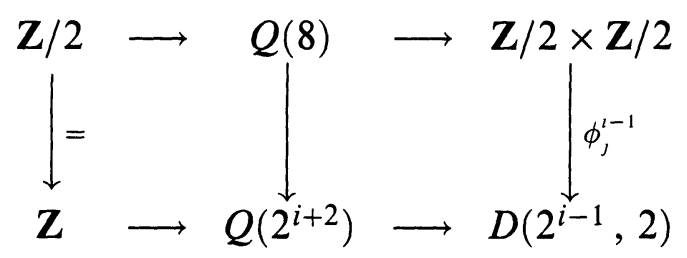

From 1.25 and its proof the $k$-invariant for the bottom extension must be $w_{i-1}+x^{2}+y^{2}$. Hence, in the spectral sequence $d^{2}(e)=$ $x^{2}+y^{2}+w_{i-1}$, so $d^{3}\left(e^{2}\right)=(x+y) w_{i-1}=x^{3}+y^{3}$ in $E_{3}^{3,0}$. But from this $d^{5}\left(e^{4}\right)=0$ and $E_{4}=E_{\infty}$. The result follows for $i>1$. The case $i=1$ is virtually the same.

In particular the quaternion groups are periodic of period 4, with

$$
H^{1}\left(Q\left(2^{i}\right) ; \mathbf{Z} / 2\right)=H^{2}\left(Q\left(2^{i}\right) ; \mathbf{Z} / 2\right)=(\mathbf{Z} / 2)^{2},
$$

and $H^{3}\left(Q\left(2^{i}\right) ; \mathbf{Z} / 2\right)=\mathbf{Z} / 2$. Moreover, the central inclusion

$$
\mathbf{Z} / 2 \longrightarrow Q\left(2^{i}\right)
$$

induces a surjection of $H_{4 *}(\mathbf{Z} / 2 ; \mathbf{Z} / 2)$ onto the periodicity classes in the homology of $Q\left(2^{i}\right)$. Explicit representatives for the classes in dimension 1 are the maps

$$
f_{1, i}: S^{1} \longrightarrow B_{Q\left(2^{i}\right)}
$$

given by surjection onto the subgroups generated by $T$, $\tau$, respectively. Similarly, explicit representatives for the classes in dimension 2 are given by the two maps

$$
f_{2, i}: M(1) \longrightarrow B_{Q\left(2^{l}\right)},
$$

surjecting $\mathbf{Z} \times_{T} \mathbf{Z}$ onto $Q\left(2^{i}\right)$, by sending $T$ to $T, \tau$ to $\tau, T \tau$, respectively. Finally, an explicit representative for the class in dimension 3 is given by the quotient $S^{3} / Q\left(2^{i}\right)$, where the action is the usual 
one. Then we have

THEOREM 1.28 .

$$
\begin{array}{r}
\Omega_{*}\left(B_{Q^{\left(2^{i}\right)}} ; \mathbf{Z} / 2\right) \\
=\bigoplus_{i \geq 2} E\left(\left\{M(i), f_{2^{i}}\right\}\right) \otimes\left\{1,\left\{S^{1}, f_{1}\right\},\left\{S^{1}, f_{2}\right\},\left\{M(1), f_{2,1}\right\},\right. \\
\left.\left\{M(1), f_{2,2}\right\},\left\{S^{3} / Q^{2^{i+2}}, f\right\}\right\}
\end{array}
$$

as a module over $\Omega_{*}\left(B_{\mathbf{Z} / 2} ; \mathbf{Z} / 2\right)$.

REMARK 1.29. Since $S^{3} / Q\left(2^{i}\right)$ is oriented its total Wu class is 1 , and all our models for generators over $\Omega_{*}(p t ; \mathbf{Z} / 2)$ have the property that all three classes $\left(V^{2}\right), V \mathrm{Sq}^{1} V,\left(\mathrm{Sq}^{1} V\right)^{2}$, are 0 in positive dimensions. As a consequence, the $\kappa_{i}, \tau_{j}, \gamma_{k}$ of the introduction in $H^{*}\left(B_{\pi} ; \mathrm{Z} / 2\right)$ for $\pi$ one of the groups above, are calculated directly from the evaluation of the images of the $\{M, f\}$ in $L_{*}^{h}\left(\mathbf{Z}\left(\zeta_{3}\right) \pi ; \mathbf{Z} / 2\right)$ without the necessity of solving complex equations involving the inversion of the $V^{2}, V \mathrm{Sq}^{1} V,\left(\mathrm{Sq}^{1} V\right)^{2}$.

2. The restriction theorem. The discussion of the boundary map $b$ in the exact sequence

$$
\begin{aligned}
\cdots & \longrightarrow \widehat{H}_{k}\left(\mathbf{Z} / 2 ; \widetilde{K}_{0}\left(\mathbf{Z}\left(\zeta_{3}\right) \pi\right)\right) \longrightarrow L_{k}^{h}\left(\mathbf{Z}\left(\zeta_{3}\right) \pi\right) \\
& \longrightarrow L_{k}^{p}\left(\mathbf{Z}\left(\zeta_{3}\right) \pi\right) \stackrel{b}{\longrightarrow} \widehat{H}_{k-1}\left(\mathbf{Z} / 2 ; \widetilde{K}_{0}\left(\mathbf{Z}\left(\zeta_{3}\right) \pi\right) \longrightarrow \cdots\right.
\end{aligned}
$$

given in $\S 6$ of [5] (in particular the paragraphs immediately preceding and following 6.2) admits a more intrinsic description. Recall that the essential tool introduced in [5] to study $\widehat{H}_{k}\left(\mathbf{Z} / 2 ; \widetilde{K}_{0}\left(\mathbf{Z}\left(\zeta_{3}\right) \pi\right)\right)$ was the exact sequence valid for an arbitrary finite 2-group

$$
\begin{aligned}
0 \longrightarrow U^{\prime} K_{1}^{\prime}\left(\mathbf{Z}\left(1 / 2, \zeta_{3}\right) \pi\right) \stackrel{p}{\longrightarrow} U K_{1}\left(\widehat{\mathbf{Q}}_{2}\left(\zeta_{3}\right) \pi\right) / K_{1}^{\prime}\left(\widehat{\mathbf{Z}}_{2}\left(\zeta_{3}\right) \pi\right) \\
\longrightarrow \widetilde{K}_{0}\left(\mathbf{Z}\left(\zeta_{3}\right) \pi\right)_{2} \longrightarrow 0 .
\end{aligned}
$$

See in particular Remark 5.8 and Corollary 5.9 there. Since

$$
\left.\widehat{H}_{*}\left(\mathbf{Z} / 2 ; U K_{1}\left(\widehat{\mathbf{Q}}_{2}\left(\zeta_{3}\right) \pi\right) / K_{1}^{\prime}\left(\widehat{\mathbf{Z}}_{2}\left(\zeta_{3}\right) \pi\right)\right)\right) \equiv 0
$$

the homology of $\widetilde{K}_{0}\left(\mathbf{Z}\left(\zeta_{3}\right) \pi\right)$ is determined by that of $U^{\prime} K_{1}^{\prime}\left(\mathbf{Z}\left(\frac{1}{2}, \zeta_{3}\right) \pi\right)$.

Denote by $b^{\prime}$ the composite map

$$
\begin{aligned}
L_{k}^{p}\left(\mathbf{Z}\left(\zeta_{3}\right) \pi\right) & \stackrel{b}{\longrightarrow} \widehat{H}_{k-1}\left(\mathbf{Z} / 2 ; \widetilde{K}_{0}\left(\mathbf{Z}\left(\zeta_{3}\right) \pi\right)\right) \\
& \cong \widehat{H}_{k}\left(\mathbf{Z} / 2 ; U^{\prime} K_{1}^{\prime}\left(\mathbf{Z}\left(1 / 2, \zeta_{3}\right) \pi\right)\right)
\end{aligned}
$$


then we have

LEMMA 2.4. (a) The determinant map $\left\langle\left[\mathbf{Z}\left(1 / 2, \zeta_{3}\right) \pi\right]^{n}, \quad B\right\rangle \rightarrow$ $K_{1}\left(\mathbf{Z}\left(1 / 2, \zeta_{3}\right) \pi\right)$, when restricted to the quadratic forms coming from $L_{k}^{p}\left(\mathbf{Z}\left(\zeta_{3}\right) \pi\right)$ induces a well defined map (the discriminant),

$$
d_{*}: L_{2 k}\left(\mathbf{Z}\left(\zeta_{3}\right) \pi\right) \longrightarrow \widehat{H}_{2 k}\left(\mathbf{Z} / 2 ; U^{\prime} K_{1}^{\prime}\left(\mathbf{Z}\left(1 / 2, \zeta_{3}\right) \pi\right)\right)
$$

and $d_{*}$ is exactly the map $b^{\prime}$ above.

(b) Let $\alpha \in L_{2 k+1}^{p}\left(\mathbf{Z}\left(\zeta_{3}\right) \pi\right)$ be represented by the formation $\left(H \oplus H^{*}, K\right)$ with

$$
0 \longrightarrow K \stackrel{p_{H}}{\longrightarrow} H^{*} \longrightarrow V \longrightarrow 0
$$

an exact sequence and $V$ an odd torsion module over $\mathbf{Z}\left(\zeta_{3}\right) \pi$. Let

$$
\left\{p_{H}\right\}=d \in U K_{1}\left(\widehat{\mathbf{Q}}_{2}\left(\zeta_{3}\right) \pi\right)
$$

represent $p_{H}$, then $\left\{d^{*} / d\right\}$ in $U K_{1}\left(\widehat{\mathbf{Q}}_{2}\left(\zeta_{3}\right) \pi\right) / K_{1}^{\prime}\left(\widehat{\mathbf{Z}}_{2}\left(\zeta_{3}\right) \pi\right)=p(a)$ for some $a$ in $U^{\prime} K_{1}^{\prime}\left(\mathbf{Z}\left(1 / 2, \zeta_{3}\right) \pi\right)$, and

$$
b^{\prime}(\alpha)=\{a\} \in H_{2 k+1}\left(\mathbf{Z} / 2 ; K_{1}^{\prime}\left(\mathbf{Z}\left(1 / 2, \zeta_{3}\right) \pi\right)\right) .
$$

$((2.4)$ is just a reformulation of the remarks in $\S 6$ of [5] already referred to.)

Since $\pi$ is a finite 2-group, all its irreducible representations arise via induction from various subquotients. Specifically, given an irreducible $\mathbf{Q} \pi$ representation, $r_{i}$, there is a subgroup $\pi_{i}$ and a surjection $p_{i}: \pi_{i} \rightarrow M_{i}$, where $M_{i}$ is a model group (i.e. one of the groups $\mathbf{Z} / 2^{j}$, $D\left(2^{i}, 2\right), Q\left(2^{i}\right)$, or $S D\left(2^{i}\right)$, \{cyclic, dihedral, quaternionic, or semidihedral, notation as in $[1]\})$. Then, $M_{i}$ has a unique faithful $\mathbf{Q}$ representation $\phi_{i}$, and

$$
r_{i}=I_{\pi_{t}}^{\pi} p_{i}^{!}\left(\phi_{i}\right)
$$

where $p_{i}^{!}$is the composition map taking representations on $M_{i}$ to representations on $\pi_{i}$, and $I_{\pi}^{\pi_{i}}$ is the induction map taking representations on $\pi_{i}$ to representations on $\pi$.

In the situation above we also have the restriction (forgetful) map $R_{\pi}^{\pi_{i}}$ which takes representations on $\pi$ and restricts them to representations on $\pi_{i}$ and the push-forward map $p_{i, !}$ taking representations on $\pi_{i}$ and pushing them forward ( $\otimes$ with $\mathbf{Q} M_{i}$ ) to representations on $M_{i}$. The induction and restriction maps are related by the Frobenius reciprocity law

$$
\left\langle r, I_{\pi_{i}}^{\pi}\left(r_{i}\right)\right\rangle=\left\langle R_{\pi}^{\pi_{t}}(r), r_{i}\right\rangle
$$


Now define the total restriction maps

$$
\begin{aligned}
& R_{\pi}: \mathbf{R}(\mathbf{Q} \pi) \longrightarrow \sum \mathbf{R}\left(\mathbf{Q} M_{i}\right), \quad \text { and } \\
& R_{\pi}: L_{*}^{d}\left(\mathbf{Z}\left(\zeta_{3}\right) \pi\right) \longrightarrow \sum L_{*}^{d}\left(\mathbf{Z}\left(\zeta_{3}\right) M_{i}\right)
\end{aligned}
$$

( $d=h$ or $p$ ) as the sum over $i$ of the compositions $p_{i, !} \circ R_{\pi^{t}}^{\pi}$, and let $p_{j}$ be projection onto the summand corresponding to $M_{j}$. As a consequence of (2.5) we have

LEMMA 2.6. Let the irreducible $\mathbf{Q}$ representations of $\pi$ be $r_{1}, \ldots$, $r_{n}$, then $p_{i} \circ R_{\pi}\left(r_{i}\right)=\phi_{i}$, and $p_{j} \circ R_{\pi}\left(r_{i}\right)=\sum n_{i, k} v_{k}$, for $j \neq i$, where the $v_{k}$ run over the non-faithful $Q$ representations of $M_{j}$. In particular $R_{\pi}$ is an injection on representation rings.

Proof. The second statement follows directly from the first. To see the first statement note that by the reciprocity law

$$
p_{i} \circ R_{\pi}\left(r_{i}\right)=\phi_{i}+\sum n_{j} v_{i, j},
$$

and we must show that all the $n_{j}$ are 0 . But the Q-rank of the representation $I_{\pi}^{\pi} p_{\pi_{i}}^{!}\left(v_{i, j}\right)$ is $\left[\pi: \pi_{i}\right] \circ\left\{\mathbf{Q}\right.$-rank $\left.v_{i, j}\right\}$, and this is strictly smaller than the Q-rank of $r_{i}=\left[\pi: \pi_{i}\right]\left\{\mathbf{Q}\right.$-rank $\left.\phi_{i}\right\}$. Consequently,

$$
\left\langle I_{\pi_{l}}^{\pi} \circ p_{i}^{!}\left(v_{i, j}\right), r_{i}\right\rangle=0,
$$

and by the reciprocity rule again $\left\langle v_{i, j}, p_{!, i} R_{\pi}\left(r_{i}\right)\right\rangle=0$. This completes the proof.

REMARK 2.7. The same result holds (with the same proof) for all fields $\mathbf{F}$ of characteristic different from 2 . Additionally, away from 2, $\mathbf{Z} \pi$ is a maximal order, and the result holds for $\mathbf{Z} \pi$ away from 2 as well. In particular, if we consider an odd order $\mathbf{Z} \pi$ torsion module $N$, we see that $N=\sum N_{i}$, one direct summand for each irreducible Q representation of $\pi, R_{\pi}(N)=\sum N$, one copy for each $i$, but after tensoring with $\mathbf{Z} M_{i}$ we get $N_{i} \oplus V$ where $V$ is a module over the $V_{i, j}$.

COROLLARY 2.8. The map $R_{\pi}: L_{*}^{p}\left(\mathbf{Z}\left(\zeta_{3}\right) \pi\right) \rightarrow \sum L_{*}^{p}\left(\mathbf{Z}\left(\zeta_{3}\right) M_{i}\right)$ is an injection.

Proof. We first verify 2.8 for the odd groups $L_{2 i+1}^{p}\left(\mathbf{Z}\left(\zeta_{3}\right) \pi\right)$. A basis for $L_{2 i+1}^{p}\left(\mathbf{Z}\left(\zeta_{3}\right) \pi\right)$ is represented by formations

$$
\alpha_{j}=\left(H \oplus H^{*}, K\right)
$$


each satisfying the hypothesis of Lemma 2.4(b). More particularly, the odd torsion module $V_{\alpha}$ is an irreducible $\mathbf{F}_{3}$ module corresponding to the irreducible representation $r_{j}$, and for $i=0$, the $r_{j}$ run over all representations induced up from $\mathbf{Z} / 2$ 's or $D\left(2^{i}, 2\right)$ 's. Similarly for $i=1$, the $r_{j}$ run over all the representations induced up from $Q\left(2^{i}\right)$ 's. Then the restrictions of these formations represent linearly independent elements in $\sum L_{2 i+1}^{p}\left(\mathbf{Z}\left(\zeta_{3}\right) M_{j}\right)$.

For $L_{2 i}^{p}\left(\mathbf{Z}\left(\zeta_{3}\right) \pi\right)$ we note that the natural map

$$
\bigotimes_{\mathbf{Q}}: L_{2 i}^{p}\left(\mathbf{Z}\left(\zeta_{3}\right) \pi\right) \longrightarrow L_{2 i}^{p}\left(\mathbf{Q}\left(\zeta_{3}\right) \pi\right)
$$

is an inclusion, so it suffices to verify the lemma for these latter groups. But

$$
L_{2 i}^{p}\left(\mathbf{Q}\left(\zeta_{3}\right) \pi\right)=\bigoplus L_{2 i}^{p}\left(M_{n_{i}}\left(\mathbf{K}_{i}\right)\right)
$$

where these $M_{n_{i}}\left(\mathbf{K}_{i}\right)$ are the irreducible representations over $\mathbf{Q}\left(\zeta_{3}\right)$. Once more the restriction and projection maps behave nicely. In particular,

$$
p_{i} \circ R: L_{2 i}^{p}\left(M_{n_{i}}\left(\mathbf{K}_{i}\right)\right) \stackrel{\cong}{\longrightarrow} L_{2 i}^{p}\left(M_{m_{i}}\left(\mathbf{K}_{i}\right)\right)
$$

where the only change is in the size of the matrices, not the field. Consequently, the lemma follows.

Extending 2.8 to the $L^{h}$ ( ) groups is not quite as direct as one would hope. The difficulty is the classes in $L_{2}^{h}\left(\mathbf{Z}\left(\zeta_{3}\right) \pi\right)$ coming from $H_{*}\left(\mathbf{Z} / 2 ; \widetilde{K}_{0}\left(\mathbf{Z}\left(\zeta_{3}\right) \pi\right)\right)$.

From $[5, \S 6]$, these classes are associated to elements coming from representations onto dihedral subquotients, and $\mathrm{Z} / 2$-subquotients. The dihedral model groups have the desired elements present in their $L^{h}$ groups, so they present little difficulty, but the $\mathbf{Z} / 2$ 's are more complex. In case the representation is a matrix ring of the form $M_{2^{i}}(\mathbf{Q})$ with $i>0$ then it is always possible to take the associated subquotient to be a dihedral group $D(4,2)$, and otherwise, the representation is pulled back by the map $\pi \longrightarrow \pi^{\mathrm{ab}}$. This reduces the problem to that for $\pi$ Abelian.

When $\pi$ is $\mathbf{Z} / 2 \times \mathbf{Z} / 2$ the element is already present as we noted in $\S 7$ of [5], so the strategy is to replace the $Z / 2$ 's by $Z / 2 \times Z / 2$ 's.

In particular, it is an easy exercise to show that for Abelian groups $\pi$ the set of maps $q_{j}: \pi \rightarrow \mathbf{Z} / 2 \times \mathbf{Z} / 2$ give an injection of the exotic elements in $L_{2}^{h}\left(\mathbf{Z}\left(\zeta_{3}\right) \pi\right)$ into the corresponding elements in the sum of the $L^{h}($ ) for these model groups. 
$R_{\pi}$ also induces maps of the algebraic $K$ groups $K_{i}(\mathbf{A} \pi) \rightarrow$ $\sum K_{i}\left(\mathbf{A} M_{i}\right)$, and in a way virtually identical to the proof above we have

LEMMA 2.9. The map $R_{\pi}: K_{1}\left(\mathbf{Z}\left(1 / 2, \zeta_{3}\right) \pi\right) \rightarrow \sum K_{1}\left(\mathbf{Z}\left(1 / 2, \zeta_{3}\right) M_{i}\right)$ is an injection.

Let us define a further map

$$
S_{\pi}: L_{k}^{h}\left(\mathbf{Z}\left(\zeta_{3}\right) \pi\right) \longrightarrow \bigoplus L_{k}^{h}\left(\mathbf{Z}\left(\zeta_{3}\right) \mathbf{Z} / 2 \times \mathbf{Z} / 2\right)
$$

where the sum above runs over all distinct homomorphisms $\pi \rightarrow \mathbf{Z} / 2 \times$ $\mathbf{Z} / 2$. 2.9, together with 2.4 implies the main technical result of this section.

\section{THEOREM 2.11. The map}

$$
R_{\pi} \oplus S: L_{*}^{h}\left(\mathbf{Z}\left(\zeta_{3}\right) \pi\right) \longrightarrow \bigoplus L_{*}^{h}\left(\mathbf{Z}\left(\zeta_{3}\right) M_{i}\right) \oplus \sum L_{*}^{h}\left(\mathbf{Z}\left(\zeta_{3}\right) \mathbf{Z} / 2 \times \mathbf{Z} / 2\right)
$$

is an injection on the subgroups described in Theorem 6.1 of [5].

Proof. The classes of interest in 6.1 all either inject into $L_{*}^{p}\left(\mathbf{Z}\left(\zeta_{3}\right) \pi\right)$ or are associated to one of the $Z / 2$ 's \{that is, the elements \pm 1 at the faithful representation of $M_{i}$ \} corresponding to $M_{i}=\mathrm{Z} / 2$, a dihedral group, or a quaternion group. So we must show that $R_{\pi}$ on such an element is not in the kernel of the map

$$
\widehat{H}_{k}\left(\mathbf{Z} / 2 ; \widetilde{K}_{0}\left(\mathbf{Z}\left(\zeta_{3}\right) M_{i}\right)\right) \longrightarrow L_{k}^{h}\left(\mathbf{Z}\left(\zeta_{3}\right) M_{i}\right) \text {. }
$$

In the case where $k$ above is even, the situation is quite clear. We consider first the case $k=3$. Then the image of $b^{\prime}$ consists exactly of the set of -1 's at the quaternion representations. In particular, the -1 's at all the other representations are not in the image of $b^{\prime}$, so the result is true.

When $k=2$ the critical elements have already been shown to exist for the dihedral restriction models and the $\mathrm{Z} / 2 \times \mathrm{Z} / 2$ models after projection to the abelianization.

When $k=1$ the image of $b^{\prime}$ consists of all the -1 's at the representations corresponding to $\mathbf{Z} / 2$ 's or dihedral groups. Once more there is no overlap onto the faithful representations for the quaternion: groups, and so the result follows in this case.

Finally, we need the case $k=0$. Here, all the elements at all the $\mathrm{Z} / 2$ and dihedral groups are hit, but the homomorphism

$$
L_{3}^{h}\left(\mathbf{Z}\left(\zeta_{3}\right) Q\left(2^{i}\right)\right) \stackrel{\tau_{K}}{\longrightarrow} L_{1}^{h}\left(\mathbf{Z} Q\left(2^{i}\right)\right)
$$


is non-trivial on the element coming from the -1 at the faithful representation, and this provides a homomorphism out, which proves the injectivity of the map at this level. Thus the proof is complete.

For later use we need the following corollary of 2.11 which reduces the calculation of the map

$$
\nu: \Omega_{k}\left(B_{\pi}\right) \longrightarrow L_{k}^{h}\left(\mathbf{Z}\left(\zeta_{3}\right) \pi\right)
$$

to the same problem but only for certain of the model groups.

Corollary 2.13. Let $a \in \Omega_{k}\left(B_{\pi}\right)$, then $\nu(a)$ is non-zero if and only if there is a $\pi_{i}$ as above so that $p_{i} \circ R_{\pi}(\nu(a))$ is non-zero. In other words, restriction to the model groups $\mathbf{Z} / 2, D\left(2^{i}, 2\right)$, or projection onto groups of the form $\mathrm{Z} / 2 \times \mathbf{Z} / 2$ completely determines the map $\nu$.

3. $L$ theory with coefficients. There is a natural orientation

$$
\hat{e}_{\zeta_{3}}: \Omega_{*}\left(B_{\pi} ; \mathbf{Z} / 2\right) \longrightarrow L_{*}^{h}\left(\mathbf{Z}\left(\zeta_{3}\right) \pi \times \mathbf{Z}^{-}\right)
$$

where the involution on $\pi \times \mathbf{Z}^{-}$is given by $\tau \leftrightarrow \tau^{-1}$ for $\tau \in \pi$, while $T \leftrightarrow-T^{-1}$ for $T$ the generator of the $\mathbf{Z}$. It is obtained by using the product

$$
f \times\left\{\hat{w}_{1}\right\}: M \longrightarrow B_{\pi} \times S^{1}
$$

where $\hat{w}: M \rightarrow S^{1}$ is the integral lifting of $w_{1}$, and $f: M \rightarrow B_{\pi}$ is the map which induces the $\pi$ covering of $M$.

For these $L$ groups we have (see e.g. [7])

THEOREM 3.2. Let $A$ be any unitary ring with involution

$$
\tau: A \longrightarrow A,
$$

and suppose $u \in \operatorname{center}(A)$ is a unit fixed under $\tau$. Define an involution

$$
\tau^{u}: A\left[t, t^{-1}\right] \longrightarrow A\left[t, t^{-1}\right]
$$

as $\tau$ on $A$ and $\tau^{u}(t)=u t^{-1}$, then there is an exact sequence

$$
\begin{gathered}
\cdots \rightarrow L_{*}^{p}(A, \tau) \stackrel{\langle 1\rangle-\langle u\rangle}{\longrightarrow} L_{*}^{h}(A, \tau) \longrightarrow L_{*}^{h}\left(A\left[t, t^{-1}\right], \tau^{u}\right) \longrightarrow \cdots \\
\cdots \longrightarrow L_{*-1}^{p}(A, \tau) \stackrel{\langle 1\rangle-\langle u\rangle}{\longrightarrow} L_{*-1}^{h}(A, \tau) \rightarrow \cdots
\end{gathered}
$$

A special case is $\tau^{-1}$, which we abbreviate as $\tau^{-}$. Consequently, the exact sequence above is extremely efficient for calculating the groups $L_{*}^{h}\left(\mathbf{Z}\left(\zeta_{3}\right) \pi \times \mathbf{Z}^{-}\right)$, but, because of the group $L_{*}^{p}\left(\mathbf{Z}\left(\zeta_{3}\right) \pi\right)$ appearing in the sequence, it is not quite optimally adapted to the study 
of the map $s_{\pi}$ of the introduction. For that we need a further class of $L$-groups.

The $L$ groups $L_{n}^{h}(A ; \mathbf{Z} / 2)$ are defined in terms of algebraic models which consist of $n$-dimensional quadratic Poincaré complexes $D$ with

$$
\partial D=\delta D \oplus \delta D
$$

where $\delta D$ is a closed Poincaré complex of dimension $n-1$. Bordism is given by an $n+1$ dimensional Poincaré complex $W$ with

$$
\partial W=D \cup_{\delta D \oplus \delta D} C \oplus C \text { where } \partial C=\delta D .
$$

There is an exact sequence

$$
\begin{aligned}
\cdots & \longrightarrow L_{n}^{h}(A) \stackrel{\times 2}{\longrightarrow} L_{n}^{h}(A) \longrightarrow L_{n}^{h}(A ; \mathbf{Z} / 2) \\
& \longrightarrow L_{n-1}^{h}(A) \stackrel{\times 2}{\longrightarrow} L_{n-1}^{h}(A) \longrightarrow \cdots
\end{aligned}
$$

and (3.1), 3.2 fit together to give the commutative diagram

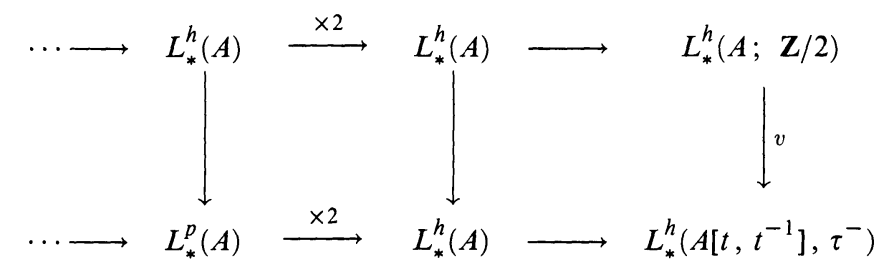

We can use the map $v$ in (3.4) to obtain information about

$$
\hat{e}_{\zeta_{3}}: \Omega_{*}\left(B_{\pi} ; \mathbf{Z} / 2\right) \longrightarrow L_{*}^{h}\left(\mathbf{Z}\left(\zeta_{3}\right) \pi ; \mathbf{Z} / 2\right)
$$

because the following diagram commutes

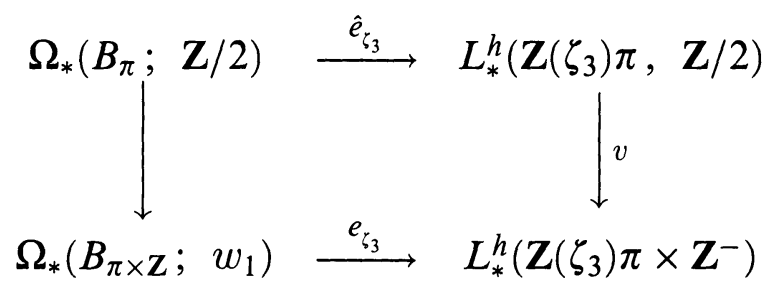

This procedure is especially effective when $\widetilde{K}_{0}\left(\mathbf{Z}\left(\zeta_{3}\right) \pi\right)=0$ which is the case when $\pi=1, \mathbf{Z} / 2$ or $\mathbf{Z} / 4$ since then $v$ is an isomorphism. The point is that standard techniques with standard closed Poincare duality chain complexes allow us to calculate the image of the map $e_{\zeta_{3}}$ above, but the map $\hat{e}_{\zeta_{3}}$ is more complex, since it requires $Z / 2$ type chain complexes, and the interpretation of the image is also more complex. This will be illustrated in the next two sections where we study the explicit images of the map $e_{\zeta_{3}}$ for $\mathrm{Z} / 2$. 
There is also a product pairing

$$
L_{h}^{i}(\mathbf{Z} ; \mathbf{Z} / 2) \otimes L_{j}^{h}\left(\mathbf{Z}\left(\zeta_{3}\right) \pi ; \mathbf{Z} / 2\right) \stackrel{\nu}{\longrightarrow} L_{i+j}^{h}\left(\mathbf{Z}\left(\zeta_{3}\right) \pi ; \mathbf{Z} / 2\right)
$$

which fits into a commutative diagram analogous to (3.5)

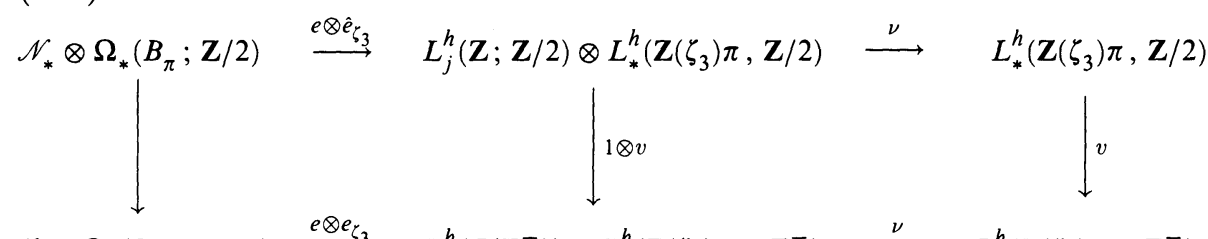

$\mathscr{N}_{*} \otimes \Omega_{*}\left(B_{\pi \times \mathbf{Z}} ; w_{1}\right) \stackrel{e \otimes \zeta_{\zeta_{3}}}{\longrightarrow} \quad L_{J}^{h}\left(\mathbf{Z}\left(\mathbf{Z}^{-}\right)\right) \otimes L_{*}^{h}\left(\mathbf{Z}\left(\zeta_{3}\right) \pi \times \mathbf{Z}^{-}\right) \stackrel{\nu}{\longrightarrow} L_{*}^{h}\left(\mathbf{Z}\left(\zeta_{3}\right) \pi \times \mathbf{Z}^{-}\right)$

We have from ([10, page 173]) that

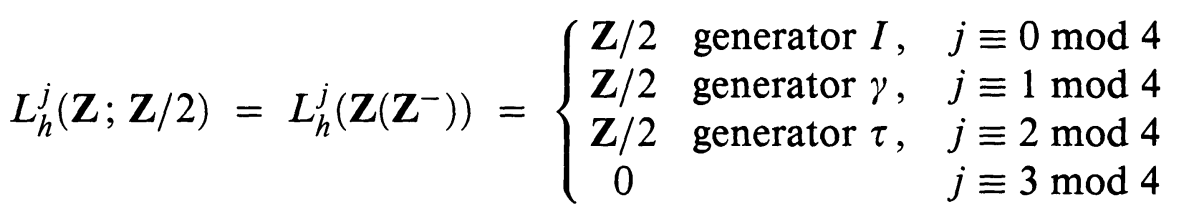

Moreover, from [3] or [9], the map

$$
\tilde{e}_{\zeta_{3}}: \mathscr{N}_{*} \longrightarrow L_{h}^{*}(\mathbf{Z})
$$

is given by the formula

$$
\tilde{e}_{\zeta_{3}}\{M\}=\left\langle V^{2},[M]\right\rangle I+\left\langle V \mathrm{Sq}^{1} V,[M]\right\rangle \gamma+\left\langle\left(\mathrm{Sq}^{1} V\right)^{2},[M]\right\rangle \tau .
$$

Thus, we obtain the general form of the evaluation formulae similar to that given in [13], as

COROLlaRy 3.8. There are well defined elements $l_{*, \kappa}, \gamma_{*, \kappa} \tau_{*, \kappa}$ in $H^{*}\left(B_{\pi} ; \mathbf{Z} / 2\right)$ which have the property that the image of $\{M, f\} \in$ $\Omega_{*}\left(B_{\pi}, \mathbf{Z} / 2\right)$ in $L_{*}^{h}\left(\mathbf{Z}\left(\zeta_{3}\right) \pi ; \mathbf{Z} / 2\right)$ is given by a collection of formulae

$$
\begin{aligned}
\left(\left\langle V^{2} f^{*}\left(l_{*, \kappa}\right),[M]\right\rangle+\left\langle V \mathrm{Sq}^{1} V f^{*}\left(\gamma_{*, \kappa}\right),[M]\right\rangle\right. & \\
& \left.+\left\langle\left(\mathrm{Sq}^{1} V\right)^{2} f^{*}\left(\tau_{*, \kappa}\right),[M]\right\rangle\right) \kappa,
\end{aligned}
$$

one for each generator $\kappa$ in $L_{*}^{h}\left(\mathbf{Z}\left(\zeta_{3}\right) \pi, \mathbf{Z} / 2\right)$.

In our explicit case it turns out that the terms involving $\gamma$ are 0 , since we have

THEOREM 3.9. The maps

$$
\begin{gathered}
e_{\zeta_{3}}(\gamma *): \Omega_{*}\left(B_{\pi}\right) \longrightarrow L_{*+1}^{h}\left(\mathbf{Z}\left(\zeta_{3}\right) \pi\right) \\
\hat{e}_{\zeta_{3}}(\gamma *): \Omega_{*}\left(B_{\pi} ; \mathbf{Z} / 2\right) \longrightarrow L_{*+1}^{h}\left(\mathbf{Z}\left(\zeta_{3}\right) \pi ; \mathbf{Z} / 2\right)
\end{gathered}
$$


are identically zero while the map

$$
e_{\zeta_{3}}(\tau *): \Omega_{*}\left(B_{\pi} ; \mathbf{Z} / 2\right) \longrightarrow L_{*+2}^{h}\left(\mathbf{Z}\left(\zeta_{3}\right) \pi ; \mathbf{Z} / 2\right)
$$

is multiplication by the non-trivial element in $L_{2}^{h}\left(\mathbf{Z}\left(\zeta_{3}\right) ; \mathbf{Z} / 2\right)=\mathbf{Z} / 2$.

Proof. $\{\gamma\}$ is represented by the formation

$$
\gamma=\left\{\begin{array}{ccc}
\mathbf{Z} & 1 & \mathbf{Z} \\
\downarrow^{2} & & \downarrow \\
\mathbf{Z} & 1 & \mathbf{Z}
\end{array}\right\}
$$

with generators $A, B$ where $\partial B=2 A$, and $\phi_{1}: B^{*} \rightarrow B$.

This can be verified by observing that the formation associated to the complex above (see [10, p. 162], for details on how to obtain this correspondence) is

$$
\left\{\mathbf{Z} \oplus \mathbf{Z},(0,1),(2,1),\left(\begin{array}{cc}
-1 & 1 \\
1 & 0
\end{array}\right)\right\}
$$

But as in ([10, pp. 169-173]) this is the boundary in the (symmetric) localization sequence of the torsion form $\{\mathbf{Z} / 2,1 / 2\}$ which represents the deRham invariant.

$\gamma \oplus \gamma$ is an explicit boundary as follows. Let $\{V, \partial, \Psi\}$ be the complex

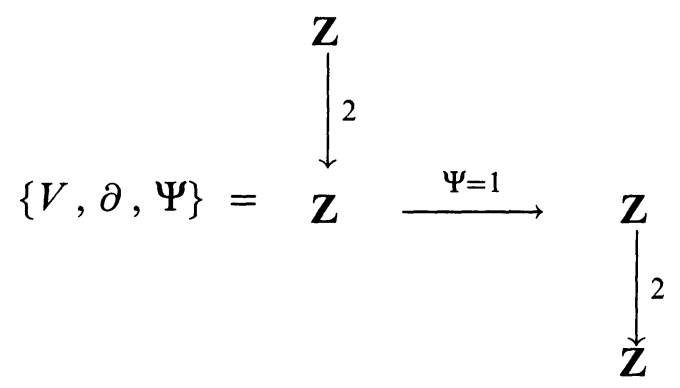

with generator $a$ in dimension $0, b$ in dimension 1 . Define

$$
f: \gamma \oplus \gamma \longrightarrow\{V, \partial, \Psi\} \text { by } f\left(A_{i}\right)=a, f\left(B_{i}\right)=b .
$$

Then we easily verify the formulae

$$
\begin{gathered}
\Psi+\Psi^{*}=f \phi_{1} f^{*}=\left\{b^{*} \mapsto 2 b\right\}, \\
\partial \Psi-\Psi \delta=\varepsilon f \phi_{0} f^{*},
\end{gathered}
$$


so $\left\{\gamma \oplus \gamma, V, f, \phi_{i}, \Psi\right\}$ is a symmetric pair. We check that it is, in fact, a Poincaré pair. Let $Y$ be the mapping cylinder of $f$, so

$$
Y=V \oplus \Sigma(\gamma \oplus \gamma) \text { with } \partial=\left(\begin{array}{cc}
\partial & 0 \\
-\varepsilon f & \partial
\end{array}\right)
$$

and consider the map

$$
\left(\phi f^{*}, \Psi\right): V^{2-*} \longrightarrow Y .
$$

It is easily checked that (3.12) is a chain homotopy equivalence, but this is the requirement that we have a Poincare pair.

Now we check the image $e_{\zeta_{3}}(\gamma)$. It is given by the diagram

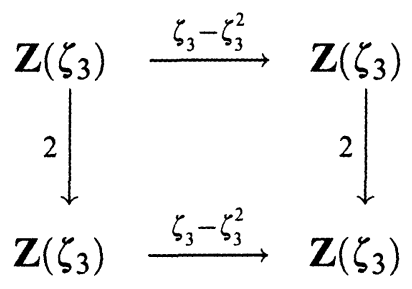

From [5] it follows that $L_{1}^{h}\left(\mathbf{Z}\left(\zeta_{3}\right)\right)=0$, so $e_{\zeta_{3}}(\gamma)$ is a boundary. Indeed, in the localization sequence the form

$$
\Psi=\left(\begin{array}{cc}
\zeta_{3}-\left(\zeta_{3}\right)^{2} & 2 \\
-2 & 2\left(\zeta_{3}-\left(\zeta_{3}\right)^{2}\right)
\end{array}\right)
$$

has boundary the torsion form $\left\langle\mathbf{F}_{4}, 1\right\rangle$. Then it is easily checked that the 2-dimensional Poincare pair $\{\partial W, W\}$ where $W=\left\{W_{1}=\right.$ $\left.\left(\mathbf{Z}\left(\zeta_{3}\right)\right)^{2}, \Psi\right\}$ has boundary $e_{\zeta_{3}}(\gamma)$.

This proves the first two statements in 3.9 , so it remains to check $\hat{e}_{\zeta_{3}}(\tau)$. Consider the union of the Poincare pairs

$$
Y^{\prime}=W \cup_{e_{\zeta_{3}}\left(\gamma_{0}\right)} e_{\zeta_{3}}(\gamma) \cup_{e_{\zeta_{3}}\left(\gamma_{1}\right)} W,
$$

(see [10, pages 135-136] for details of this construction). This is a closed Poincaré complex in $L_{2}^{h}\left(Z\left(\zeta_{3}\right)\right)$, and its image in $L_{2}^{h}\left(\mathbf{Z}\left(\zeta_{3}\right) ; \mathbf{Z} / 2\right)$ represents $\hat{e}_{\zeta_{3}}(\tau)$. On the other hand,

$$
\mathbf{Z}=L_{2}^{h}\left(\mathbf{Z}\left(\zeta_{3}\right)\right) \longrightarrow L_{2}^{h}\left(\mathbf{Q}\left(\zeta_{3}\right)\right)
$$

is an injection, so, in order to evaluate the class of $Y^{\prime}$ in $L_{2}^{h}\left(Z\left(\zeta_{3}\right)\right)$ we need only evaluate its image in $L_{2}^{h}\left(\mathbf{Q}\left(\zeta_{3}\right)\right)$. But

$$
Y^{\prime} \otimes \mathbf{Q} \sim W \otimes \mathbf{Q} \oplus W \otimes \mathbf{Q},
$$

since $e_{\zeta_{3}}(\gamma)$ and $e_{\zeta_{3}}()$ are rationally trivial. 
From the definition of $\{W, \Psi\}$ it follows that

$$
W \otimes \mathbf{Q}=\left\{\mathbf{Q}\left(\zeta_{3}\right) \oplus \mathbf{Q}\left(\zeta_{3}\right), \Psi\right\}
$$

diagonalizes to

$$
\left(\begin{array}{cc}
\zeta_{3}-\left(\zeta_{3}\right)^{2} & 0 \\
0 & 2\left(\zeta_{3}-\left(\zeta_{3}\right)^{2}\right)
\end{array}\right)
$$

in $L_{2}^{h}\left(\mathbf{Q}\left(\zeta_{3}\right)\right)$, and twice this is the image of the generator of $L_{2}^{h}\left(\mathbf{Z}\left(\zeta_{3}\right)\right)$. The proof of 3.9 is complete.

REMARK 3.14. In particular 3.9 implies that the only coefficients appearing in the general product formulae have the form $\left\langle(V)^{2} f^{*}(\kappa)\right.$, $[M]\rangle$ or $\left\langle\left(\mathrm{Sq}^{1} V\right)^{2} f^{*}\left(\kappa^{\prime}\right),[M]\right\rangle$. The first terms are unavoidable, but the second terms are highly undesirable. For our model groups we will return to these second terms in $\S 6$.

4. The products $S^{1} \times_{T} \mathbf{R} \mathbf{P}^{4 n-1}$. The fundamental group of $S^{1} \times_{T}$ $\mathbf{R P}^{4 n-1}$ is $\mathbf{Z} \times \mathbf{Z} / 2=\left\{\tau, T \mid T^{2}=1, T \tau=\tau T\right\}$ and the associated involution of the group ring $\mathbf{Z}(\mathbf{Z} \times \mathbf{Z} / 2)$ is generated by $\tau \leftrightarrow-\tau^{-1}$, $T \leftrightarrow T$, moreover its universal cover is $\mathbf{R} \times S^{4 n-1}$.

An equivariant cell decomposition is given as the product of the usual decomposition of $S^{4 n-1}$ into + and - hemispheres and the line into the union of its integer points and the intervals $[m, m+1]$, as $m$ runs over the integers. Moreover, on cells of the form $e_{i} \otimes e_{j}$, the operation of the fundamental group is just the product action as long as $j \neq 4 n-1$, but for $j=4 n-1$ we have

$$
T\left(e_{i} \times e_{4 n-1}^{+}\right)=e_{i} \times e_{4 n-1}^{-} \text {, while } \tau\left(e_{i} \otimes e_{4 n-1}^{+}\right)=-\tau\left(e_{i}\right) \times e_{4 n-1}^{-} \text {. }
$$

Our object in this section is to study the class of the image $\left\langle\mathbf{Z},-\left(\zeta_{3}\right)\right\rangle$ $\otimes\{\mathscr{C}, \phi\}$ in $L_{0}^{h}\left(\mathbf{Z}\left(\zeta_{3}\right) \mathbf{Z} / 2 ; \mathbf{Z} / 2\right)$, where $\{\mathscr{C}, \phi\}$ is the Ranicki symmetric structure induced on the complex for $S^{1} \times_{T} \mathbf{R} \mathbf{P}^{4 n-1}$ via a suitable diagonal approximation to the lifting of the geometric diagonal map

$$
\widehat{\Delta}: S^{1} \times_{T} \mathbf{R} \mathbf{P}^{4 n-1} \longrightarrow\left(\mathbf{R} \times S^{4 n-1}\right) \times_{\mathbf{Z} / 2 \times \mathbf{Z}}\left(\mathbf{R} \times S^{4 n-1}\right) .
$$

Specifically, since the first Stiefel-Whitney class of $S^{1} \times_{T} \mathbf{R} \mathbf{P}^{4 n-1}$ is non-trivial, the generating chain associated to the chain map $\mathscr{C}^{4 n-*} \longrightarrow$ $\mathscr{C}_{*}$ actually occurs in the complex $\left(\mathscr{C}_{*} \otimes \mathscr{C}_{*}\right) \otimes \mathbf{Z}(\mathbf{Z} \times \mathbf{Z} / 2) \mathbf{Z}^{\phi}$ where $\mathbf{Z}^{\phi}$ is the $\mathbf{Z}(\mathbf{Z} \times \mathbf{Z} / 2)$ module induced by the homomorphism $\phi: \mathbf{Z} \times \mathbf{Z} / 2 \longrightarrow \mathbf{Z} / 2$ associated to the the first Stiefel-Whitney class. 
But it is explicitly given by taking the usual equivariant diagonal approximation

$$
\widetilde{\Delta}: \mathscr{C}_{*} \longrightarrow \mathscr{C}_{*} \otimes \mathscr{C}_{*},
$$

tensoring it with $\mathbf{Z}^{\phi}$, and taking the image of the cycle associated to a generator of the homology group

$$
H_{4 n}\left(\mathscr{C}_{*} \otimes_{\mathbf{Z}(\mathbf{Z} \times \mathbf{Z} / 2)} \mathbf{Z}^{\phi}\right)=\mathbf{Z} .
$$

Recall, that an equivariant diagonal approximation on $\mathscr{C}\left(S^{4 n-1}\right)$ is given by the formla

$$
\widehat{\Delta}_{\#}\left(e_{i}^{+}\right)=\sum e_{j} \otimes\left(T^{j}\right) e_{i-j} .
$$

(see e.g. [11] for details), and a Z-equivariant diagonal approximation on $\mathbf{R}$ is given by

$$
e_{0} \longrightarrow e_{0} \otimes e_{0}, e_{1} \longrightarrow e_{0} \otimes e_{1}+e_{1} \otimes \tau e_{0} .
$$

On tensoring these maps together we get a suitable diagonal approximation on the $4 n-2$-skeleton. It remains to extend these maps over the $4 n-1$ and $4 n$ skeletons.

On the $4 n-1$ skeleton everything is defined except for the cell $e_{0} \times e_{4 n-1}^{+}$. But on this cell the previous diagonal approximation preserves the boundary, so extend the map by equivariance to its translates under $\mathbf{Z} \times \mathbf{Z} / 2$. The carrier of the image is correct (and acyclic), hence the map is correct on the $4 n-1$ skeleton.

The $4 n$ skeleton is a single copy of $\mathbf{Z}(\mathbf{Z} \times \mathbf{Z} / 2)$ with generator $e_{1} \times e_{4 n-1}^{+}$, and a long but direct calculation gives

$$
\begin{aligned}
\Delta\left(\partial e_{1} \times e_{4 n-1}^{+}\right) & \\
=\partial\left\{1 \times T \tau\left(e_{1} \times e_{4 n-1}^{+} \otimes 1\right)+1 \otimes e_{1} \times e_{4 n-1}^{+}\right. & \\
& +\sum_{n-1 \geq i \geq 1} e_{1} \times e_{i} \otimes T^{i} \tau \varepsilon_{0} \times e_{4 n-i-1} \\
& +\sum_{(-1)^{i} e_{0} \times e_{i} \otimes T^{i} e_{1} \times e_{4 n-i-1}} \\
& -\sum_{0 \leq i<n} \tau e_{0} \times e_{i} \otimes \tau T^{i} e_{0} \times e_{4 n-i-1}-e_{1} \times e_{0} \otimes T \tau e_{0} \times e_{4 n-1}^{+} \\
& \left.-e_{0} \times e_{4 n-1}^{+} \otimes T e_{1} \times e_{0}+\tau T \times \tau T\left(e_{0} \times e_{4 n-1}^{+} \otimes e_{0} \times e_{1}\right)\right\} .
\end{aligned}
$$

Once more, the class above has the correct acyclic carrier, hence provides (after extending equivariantly) a suitable diagonal approximation. 
THEOREM 4.4. The class of

$$
\left\{\mathscr{C}^{4 n-*} \rightarrow \mathscr{C}_{*}\right\} \otimes\left\langle 1, \zeta_{3}\right\rangle
$$

in $L_{4 n}^{h}\left(\mathbf{Z}\left(\zeta_{3}\right) \mathbf{Z}^{-} \times \mathbf{Z} / 2\right)$ is the image of the class $\langle 1\rangle \perp\langle T\rangle$ coming from $L_{4 n}^{h}\left(\mathbf{Z}\left(\zeta_{3}\right) \mathbf{Z} / 2\right)$ under the usual inclusion.

Proof. After tensoring with $\mathbf{Z}^{\phi}$ the chain above gives the map $\phi$ as follows:

(0) $\left(e_{1} \times e_{4 n-1}^{+}\right)^{*} \longrightarrow T \tau\left(e_{0} \times e_{0}\right)$

(1) $\left(e_{1} \times e_{4 n-2}\right)^{*} \longrightarrow-\tau e_{0} \times e_{1}$

$\left(e_{0} \times e_{4 n-1}\right)^{*} \longrightarrow T\left(e_{1} \times e_{0}\right)+e_{0} \times e_{1}$

(2) $\left(e_{1} \times e_{4 n-3}\right)^{*} \longrightarrow-e_{0} \times T \tau e_{2}$

$\left(e_{0} \times e_{4 n-2}\right)^{*} \longrightarrow-e_{1} \times e_{1}-e_{0} \times e_{2}$

(3) $\left(e_{1} \times e_{4 n-4}\right)^{*} \longrightarrow-e_{0} \times \tau e_{3}$

$\left(e_{0} \times e_{4 n-3}\right)^{*} \longrightarrow T e_{1} \times e_{2}-T e_{0} \times e_{3}$

(4) $\left(e_{1} \times e_{4 n-5}\right)^{*} \longrightarrow e_{0} \times T \tau e_{4}$

$\left(e_{0} \times e_{4 n-4}\right)^{*} \longrightarrow e_{1} \times e_{3}+e_{0} \times e_{4}$

(5) $\left(e_{1} \times e_{4 n-6}\right)^{*} \longrightarrow e_{0} \times \tau e_{5}$

$\left(e_{0} \times e_{4 n-5}\right)^{*} \longrightarrow-T\left(e_{1} \times e_{4}\right)+T\left(e_{0} \times e_{5}\right)$

and from here on it is periodic. Thus we have the array

$$
\begin{array}{ccccc} 
& e_{0} \times 22 n & e_{1} \times e_{2 n-1} & \left(e_{1} \times e_{2 n}\right)^{*} & \left(e_{0} \times e_{2 n+1}\right)^{*} \\
\left(e_{0} \times e_{2 n}\right)^{*} & 1 & -\zeta+\tau^{-1} T \zeta^{2} & -\left(\tau^{-1}+1\right) & T-1 \\
\left(e_{1} \times e_{2 n-1}\right)^{*} & -T \tau \zeta-\zeta^{2} & 0 & -(T+1) & 0 \\
e_{1} \times e_{2 n} & \tau-1 & -(T+1) & 0 & 0 \\
e_{0} \times e_{2 n+1} & T-1 & 0 & 0 & 0
\end{array}
$$

Do row reduction so as to replace the second row by the sum

$$
R_{2}+\zeta \tau R_{4}+\zeta R_{3}=R_{2}^{\prime}
$$

Then the image of $R_{2}$ is replaced by

$$
(1,-\zeta(T+1),-(T+1), 0)
$$

and it is easily checked that $R_{2}^{\prime}$ and $R_{1}$ map onto generators for the image. Thus we choose $\left\langle R_{2}^{\prime}, R_{1}\right\rangle$ as a basis for the free complex and calculate the resulting non-singular form as

$$
\left(\begin{array}{cc}
1 & 1 \\
1 & 1+T
\end{array}\right)
$$

But this is equivalent to

$$
\left(\begin{array}{ll}
1 & 0 \\
0 & T
\end{array}\right)
$$

and the result follows. 
5. The case $\mathbf{Z} \times_{T} \mathbf{Z} . \mathbf{Z} \times_{T} \mathbf{Z}=\left\{T, \tau \mid \tau^{-1} T \tau T=1\right\}$, and a resolution is given by the complex

$$
C[A] \longrightarrow C[e] \oplus C[f] \longrightarrow C[1]
$$

where

$$
\begin{aligned}
& \partial[e]=(T-1)[1] \\
& \partial[f]=(\tau-1)[1] \\
& \partial[A]=\left(T^{-1}+\tau^{-1}\right)[e]+\left(\tau^{-1} T-\tau^{-1}\right)[f]
\end{aligned}
$$

We define an involution on the group ring $\mathbf{Z}\left(\mathbf{Z} \times{ }_{T} \mathbf{Z}\right)$ by $T \leftrightarrow T^{-1}$, $\tau \leftrightarrow\left(-\tau^{-1}\right)$. Then we have

Proposition 5.3. Given a homomorphism $\lambda: \mathbf{Z} \times_{* T} \mathbf{Z} \rightarrow \pi$, there is an involution preserving homomorphism

$$
\hat{\lambda}: \mathbf{Z} \times_{T} \mathbf{Z} \longrightarrow \pi \times \mathbf{Z}^{-},
$$

which, on projection to $\pi$ is just $\lambda$.

(Indeed, $\mathbf{Z} \times_{T} \mathbf{Z} /\left[\mathbf{Z} \times_{T} \mathbf{Z}, \mathbf{Z} \times_{T} \mathbf{Z}\right]=\mathbf{Z}$ and the projection $p$ : $\mathbf{Z} \times_{T} \mathbf{Z} \rightarrow \mathbf{Z}$, of $\mathbf{Z} \times_{T} \mathbf{Z}$ onto its abelianization is involution preserving provided $\mathbf{Z}$ is given the involution $\tau \leftrightarrow-\tau^{-1}$. Then the map $\hat{\lambda}$ is the product $\lambda \times p$, and it is direct to see that it preserves involutions.)

We now describe the explicit quadratic form associated to this group, resolution, and involution. With respect to the involution, the dual complex is

$$
C[1]^{*} \longrightarrow C[e]^{*} \oplus C[f]^{*} \longrightarrow C[A]^{*}
$$

where

$$
\begin{aligned}
& \delta[e]^{*}=(T-\tau)[A]^{*}, \\
& \delta[f]^{*}=\left(-T^{-1} \tau+\tau\right)[A]^{*}, \\
& \delta[1]^{*}=\left(T^{-1}-1\right)[e]^{*}-\left(\tau^{-1}+1\right)[f]^{*} .
\end{aligned}
$$

A chain equivalence $\phi: C^{2-*} \rightarrow C_{*}$ homotopic to the equivalence induced from the geometric diagonal approximation (see the remarks preceding $4.1,4.2$ in $\S 4$ ) is given by the formulae

$$
\begin{aligned}
& {[A]^{*} \longrightarrow[1]} \\
& {[e]^{*} \longrightarrow[e]-[f]} \\
& {[f]^{*} \longrightarrow\left(1-T^{-1}\right)[f]+T^{-1}[e]} \\
& {[1]^{*} \longrightarrow-T[A] .}
\end{aligned}
$$


In the mapping cone of $\phi_{*}$, when we look at the boundary map from dimension 2 to dimension 1 we get the array

$[e]$

$[e]^{*} \quad \zeta-\zeta^{2}$

$[f]^{*} \quad \zeta T^{-1}+\zeta^{2}$

[A] $\left(\tau^{-1}+T^{-1}\right)$ $[f]$

$-\left(\zeta+T \zeta^{2}\right)$

$\zeta-\zeta^{2}+\zeta^{2} T-\zeta T^{-1}$

$\tau^{-1} T-\tau^{-1}$
$[A]^{*}$

$-T+\tau$

$T^{-1} \tau-\tau$

0

When we apply row reduction to the matrix above we find that the kernel in dimension one of the boundary map $\partial_{1}$ is two copies of $\mathbf{Z}\left(\mathbf{Z} \times{ }_{T} \mathbf{Z}\right)$, and it is the image of $\partial_{2}$. Moreover, two generators which surject onto $\operatorname{ker}\left(\partial_{1}\right)$ can be chosen as

$$
(1-T)[e]^{*}-T[f]^{*} \longrightarrow\left(T-\zeta^{2}(1-T)\right)[e]+T(1-T)[A]^{*}
$$

and

$$
[e]^{*}+[f]^{*}-\zeta T^{-1} \tau[A] \longrightarrow \zeta\left(1-T^{-2} \tau\right)[e]+f+\left(\tau T^{-1}-T\right)[A]^{*} .
$$

From this we can easily calculate the matrix of the associated skew symmetric quadratic form as

$$
Q_{K}=\left(\begin{array}{cc}
\zeta^{2}\left(T^{-1}-1\right)-\zeta(T-1) & T+\zeta^{2}\left(1+T^{2} \tau^{-1}\right)(T-1) \\
-T^{-1}-\zeta\left(1-T^{-2} \tau\right)\left(T^{-1}-1\right) & -\left(\zeta T^{-2} \tau+\zeta^{2} T^{2} \tau^{-1}\right)
\end{array}\right)
$$

(Here $Q_{K}$ denotes the invariant for the Klein bottle.) Now, consider the surjection

$$
\nu: \mathbf{Z} \times_{T} \mathbf{Z} \longrightarrow \mathbf{Z} / 2
$$

defined by $\nu(T)=T, \nu(\tau)=1$. As above, this gives the involution preserving homomorphism

$$
\hat{\nu}: \mathbf{Z} \times_{T} \mathbf{Z} \longrightarrow \mathbf{Z} / 2 \times \mathbf{Z}^{-},
$$

and we have

$$
\text { (5.7) } \hat{\nu}\left(Q_{K}\right)=\left(\begin{array}{cc}
\left(\zeta^{2}-\zeta\right)(T-1) & T+\zeta^{2}\left(1+\tau^{-1}\right)(T-1) \\
-T-\zeta(1-\tau)(T-1) & -\left(\zeta \tau+\zeta^{2} \tau^{-1}\right)
\end{array}\right) .
$$

However, the following equivalent form will be more useful to us $\hat{\nu}\left(Q_{K}\right) \sim$

$$
\begin{aligned}
\left(\begin{array}{cc}
1 & 0 \\
\zeta T \tau & 1
\end{array}\right)\left(\begin{array}{cr}
\left(\zeta^{2}-\zeta\right)(T-1) & T+\zeta^{2}\left(1+\tau^{-1}\right)(T-1) \\
-T-\zeta(1-\tau)(T-1) & -\left(\zeta \tau+\zeta^{2} \tau^{-1}\right)
\end{array}\right) & \\
& \cdot\left(\begin{array}{cc}
1 & -\zeta^{2} T \tau^{-1} \\
0 & 1
\end{array}\right) \\
\quad & \left(\begin{array}{cc}
\left(\zeta^{2}-\zeta\right)(T-1) & T+\left(\zeta^{2}+2 \tau^{-1}\right)(T-1) \\
-T-(\zeta+2 \tau)(T-1) & -\left(\tau+\tau^{-1}+\zeta^{2}-\zeta\right)(T-1)
\end{array}\right) .
\end{aligned}
$$


In particular we have

Proposition 5.8. $\langle T\rangle \otimes \hat{\nu}\left(Q_{K}\right) \sim\langle-1\rangle \otimes \hat{\nu}\left(Q_{K}\right)$.

(Indeed, using the conjugate form of $\hat{\nu}\left(Q_{K}\right)$ given above we see that

$$
(\langle 1\rangle \oplus\langle T\rangle) \otimes \hat{\nu}\left(Q_{K}\right)
$$

has a kernel with a basis of the form

$$
\left(\langle 1\rangle \otimes e_{1},\langle T\rangle \otimes e_{1}\right), \quad\left(\langle 1\rangle \otimes e_{2},\langle T\rangle \otimes(-T) e_{2}\right),
$$

and the result follows.)

Finally, we need to point out the result of using the product in $\Omega_{*}\left(B_{\mathbf{Z} / 2} ; \mathbf{Z} / 2\right)$, at least as regards its effect on the map $\lambda$.

COROLlaRY 5.9. The product map

$$
\gamma:\left\{S^{1} \times_{T} S^{1}\right\} \times\left\{S^{1} \times_{T} S^{1}\right\} \rightarrow B_{\mathbf{Z} / 2} \times B_{\mathbf{Z} / 2}=B_{\mathbf{Z} / 2 \times \mathbf{Z} / 2}
$$

is represented in $L_{0}^{h}\left(\mathbf{Z}\left(\zeta_{3}\right) \mathbf{Z} / 2 \times \mathbf{Z} / 2 ; \mathbf{Z} / 2\right)$ either by 0 or by the image of the class $\langle 1\rangle \perp\left\langle T_{1}\right\rangle \perp\left\langle T_{2}\right\rangle \perp\left\langle T_{1} T_{2}\right\rangle$ in $L_{0}^{h}\left(\mathbf{Z}\left(\zeta_{3}\right) \mathbf{Z} / 2 \times \mathbf{Z} / 2\right)$.

Proof. Denote the product map $B_{\mathrm{Z} / 2} \times B_{\mathrm{Z} / 2} \rightarrow B_{\mathrm{Z} / 2}$ by $p$, then the composite map $p \gamma$ is null cobordant as an element in the bordism group $\Omega_{4}\left(B_{\mathbf{Z} / 2} ; \mathbf{Z} / 2\right)$. Consequently, $e_{\zeta_{3}}(p \gamma)$ is also 0 in $L_{0}^{h}\left(\mathbf{Z}\left(\zeta_{3}\right) \mathbf{Z} / 2 ; \mathbf{Z} / 2\right)$. But, at the same time the class of $\left\{S^{1} \times_{T} S^{1}\right\}$ in $\Omega_{*}(p t ; \mathbf{Z} / 2)$ is also 0 . Thus all three non-trivial projections $B_{\mathbf{Z} / 2 \times \mathbf{Z} / 2} \rightarrow B_{\mathbf{Z} / 2}$ take $e_{\zeta_{3}}(\gamma)$ to 0 . Now, from [5], we know that $L_{3}^{h}\left(\mathbf{Z}\left(\zeta_{3}\right) \mathbf{Z} / 2 \times \mathbf{Z} / 2\right)=0$, while $L_{0}^{h}\left(\mathbf{Z}\left(\zeta_{3}\right) \mathbf{Z} / 2 \times \mathbf{Z} / 2\right)=\mathbf{Z}^{4}$ with generators $\langle 1\rangle,\langle 1\rangle \perp\left\langle T_{1}\right\rangle,\langle 1\rangle \perp\left\langle T_{2}\right\rangle$, and $\langle 1\rangle \perp\left\langle T_{1}\right\rangle \perp\left\langle T_{2}\right\rangle \perp\left\langle T_{1} T_{2}\right\rangle$. But it is direct to check that after tensoring with $Z / 2$, the intersection of the kernels of the various projections is a single copy of $\mathbf{Z} / 2$ generated by the element in the corollary. The result follows.

\section{The proof of the main theorems.}

Lemma 6.1. Let $\left(\mathscr{C}, \phi_{*}\right),\left(\mathscr{D}, \phi_{*}^{\prime}\right)$ be two symmetric Poincaré $d u$ ality complexes of even dimensions $2 n, 2 m$ respectively. Then

$$
e_{\zeta_{3}}\left(\mathscr{C} \otimes \mathscr{D}, \phi_{*} \otimes \phi_{*}^{\prime}\right)=-g\left(e_{\zeta_{3}}\left(\mathscr{C}, \phi_{*}\right) \otimes e_{\zeta_{3}}\left(\Delta, \phi_{*}^{\prime}\right)\right)
$$

where $g: \mathbf{Z}\left(\zeta_{3}\right) \rightarrow \mathbf{Z}\left(\zeta_{3}\right)$ is the Galois automorphism.

(The proof is direct. The associated class for $e_{\zeta_{3}}\left(\mathscr{C}, \phi_{*}\right)$ is $\left(\mathscr{C},-\phi_{*} \zeta_{3}\right)$, and similarly for $\mathscr{D}$. When we tensor them together 
we get the complex $\left(\mathscr{C} \otimes \mathscr{D}, \phi_{*} \otimes \phi_{*}^{\prime}\left(\zeta_{3}\right)^{2}\right)$. Now, apply the instant surgery construction, as was done in $\S 4$ and $\S 5$. It is easily checked that when the dimensions are both even, exchanging $-\zeta_{3}$ for $\left(\zeta_{3}\right)^{2}$ has the effect of exchanging $\left(\zeta_{3}\right)^{2}$ for $\zeta_{3}$ and switches the signs on all the terms in the matrix of the associated non-singular quadratic form.)

We now consider the case of $B_{\mathbf{Z} / 2}$. From $\S 1$ we need only consider the images $e_{\zeta_{3}}\left(S^{1}\right), e_{\zeta_{3}}\left(S^{1} \times_{T} S^{1}\right), e_{\zeta_{3}}\left(S^{1} \times_{T} \mathbf{R P}^{3}\right), \ldots, e_{\zeta_{3}}\left(S^{1} \times_{T} \mathbf{R} \mathbf{P}^{2^{\prime}-1}\right) \cdots$, and we have

LEMMA 6.2. Each of the generators above has non-trivial image in $L_{0}^{h}\left(\mathbf{Z}\left(\zeta_{3}\right) \mathbf{Z} / 2 ; \mathbf{Z} / 2\right)$, but every product maps to 0 .

Proof. The results of $\S 4$ and $\S 5$ imply the non-triviality of the images of the generators. Moreover, from 4.4 we have that $e_{\zeta_{3}}(M(i))$ is the image of $\langle 1\rangle \perp\langle T\rangle$ for $i>1$. Now, 5.8, 5.9 show that the product of the generator in dimension 2 with any of the above generators gives 0 in $L_{2}^{h}\left(\mathbf{Z}\left(\zeta_{3}\right) \mathbf{Z} / 2 ; \mathbf{Z} / 2\right)$. Likewise, the product

$$
(\langle 1\rangle \perp\langle T\rangle)^{2}=\langle 1\rangle \perp\langle 1\rangle \perp\langle T\rangle \perp\langle T\rangle,
$$

and this certainly goes to 0 in $L_{2}^{h}\left(\mathbf{Z}\left(\zeta_{3}\right) \mathbf{Z} / 2 ; \mathbf{Z} / 2\right)$. Finally, the results of [5] give that $(\langle 1\rangle \perp\langle T\rangle) \otimes e_{\zeta_{3}}\left(S^{1}\right)=0$, and 6.2 follows.

COROLlaRY 6.3. The $\kappa$ 's for $B_{\mathrm{Z} / 2}$ are non-zero precisely when the dimension has the form $2^{i}$, for $i=0,1,2, \ldots$.

(Since the model manifolds have trivial Wu-product classes $V^{2}$, $\sum V_{i} \mathrm{Sq}^{1}\left(V_{i}\right), \quad\left(\mathrm{Sq}^{1} V\right)^{2}$ it follows that the homomorphism into $L_{2}^{h}\left(\mathbf{Z}\left(\zeta_{3}\right) Z / 2 ; \mathbf{Z} / 2\right)$ directly calculates the $\kappa$ 's. See e.g. [13], [3], [14], and especially Theorem 3.9.)

There are some additional difficulties for the group $G=\mathbf{Z} / 2 \times \mathbf{Z} / 2$.

LEMMA 6.4. (a) $e_{\zeta_{3}}(M(i) \times 1), e_{\zeta_{3}}(1 \times M(i)), e_{\zeta_{3}}(M(0) \times M(0))$ are all non-zero.

(b) $e_{\zeta_{3}}(M(i) \times M(j))=e_{\zeta_{3}}(M(j) \times M(i))$ all $i, j$.

(c) $e_{\zeta_{3}}(M(0) \times M(i))=0$ for $i>1$, while $e_{\zeta_{3}}(M(i) \times M(j))=$ $\langle 1\rangle \perp\left\langle T_{1}\right\rangle \perp\left\langle T_{2}\right\rangle \perp\left\langle T_{1} T_{2}\right\rangle=V \neq 0$ for $i, j>1$.

(d) $e_{\zeta_{3}}(M(1) \times M(1))$ is either 0 or $V$. 
Proof. (a) $L_{2}^{h}\left(\mathbf{Z}\left(\zeta_{3}\right) G\right)=\mathbf{Z} / 2 \oplus \mathbf{Z}^{4}$ and in [7], [5] we showed that $S^{1} \times S^{1}$ represents the non-trivial torsion element in this dimension. Likewise, to see the rest of (a), use the projection $B_{\mathbf{Z} / 2 \times \mathbf{Z} / 2} \rightarrow B_{\mathbf{Z} / 2}$.

(b) From 6.2 the composite

$$
p_{i}\left(e_{\zeta_{3}}(M(i) \times M(j))\right) \in L_{*}^{h}\left(\mathbf{Z}\left(\zeta_{3}\right) \mathbf{Z} / 2 ; \mathbf{Z} / 2\right)=0
$$

for each of the three projections $\mathbf{Z} / 2 \times \mathbf{Z} / 2 \rightarrow \mathbf{Z} / 2$. Moreover, in dimension 0 , the intersection of the three kernels is generated by $V$ (in $L_{0}^{h}\left(\mathbf{Z}\left(\zeta_{3}\right) \mathbf{Z} / 2\right)$ we have that $p_{i}(V)=2 X_{i}$ for each $\left.p_{i}\right)$, while in dimension 2 the intersection of the kernels is the image of the torsion element in $L_{2}^{h}\left(\mathbf{Z}\left(\zeta_{3}\right) G\right)$.

(c) The symmetric complex associated to $S^{1}\left(\pi_{1}=\mathbf{Z}\right)$ is

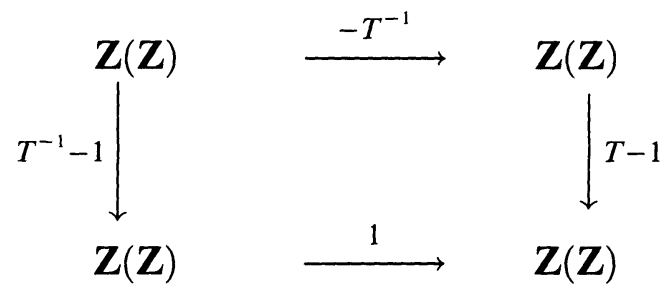

Its product with $-\zeta_{3} T_{2}$ gives the formation (over $G$ )

$$
\left(\mathbf{Z} G \oplus \mathbf{Z} G, K=\mathbf{Z} G\left\langle T_{1}-1, \zeta_{3} T_{1} T_{2}+\left(\zeta_{3}\right)^{2} T_{2}\right\rangle\right) .
$$

Similarly, the product of (6.5) with $-\zeta_{3}$ gives the formation

$$
\left(\mathbf{Z} G \oplus \mathbf{Z} G, K=\mathbf{Z} G\left\langle T_{1}-1, \zeta T_{1}^{-1}+\left(\zeta_{3}\right)^{2}\right\rangle\right) .
$$

In each case projection to the second factor gives $F_{3}^{-+} \oplus F_{3}^{--}$as quotient. Moreover, the induced torsion forms on these two modules are isomorphic, so standard local-global arguments show that their sum is equivalent to a torsion form with kernel $F_{3}^{-+} \oplus F_{3}^{--}$which has Euler characteristic zero. This implies $e_{\zeta_{3}}(M(i) \times M(0))=0$. On the other hand 6.1, 4.5 show that $e_{\zeta_{3}}(M(i) \times M(j))=V$ for $i, j>1$.

d) follows as in the proof of (b).

For $D\left(2^{i}, 2\right)$ we have

LEMMA 6.8. The maps $\phi_{j}^{i}: \mathbf{Z} / 2 \times \mathbf{Z} / 2 \rightarrow D\left(2^{i}\right.$, 2) satisfy $\left(\phi_{j}^{i}\right)(V) \neq$ 0 in the groups

$$
L_{0}^{h}\left(\mathbf{Z}\left(\zeta_{3}\right) D\left(2^{i}, 2\right) ; \mathbf{Z} / 2\right) .
$$

Proof. The ring $\mathbf{Q}\left(D\left(2^{i}, 2\right)\right)$ splits as $M_{2}\left(\mathbf{Q}\left(\lambda_{i}\right)\right) \oplus M_{2}\left(\mathbf{Q}\left(\lambda_{i-1}\right)\right) \oplus \cdots \oplus M_{2}(\mathbf{Q}) \oplus \mathbf{Q}^{++} \oplus \mathbf{Q}^{+-} \oplus \mathbf{Q}^{-+} \oplus \mathbf{Q}^{--}$, 
where $\lambda_{j}=\zeta_{2^{j}}+\left(\zeta_{2^{j}}\right)^{-1}$, and $M_{2}(\mathbf{F})$ is the 2-matrix ring with coefficients in $\mathbf{F}$. Let $I$ be the induction map from the representation ring for $G$ to that for $D\left(2^{i}, 2\right)$, and let $V^{j}$ be the irreducible representation associated to the component $M_{2}\left(\mathbf{Q}\left(\lambda_{j}\right)\right)$ above, then we have

$$
\begin{aligned}
& I\left(\mathbf{Q}^{-\varepsilon}\right)=V^{i} \\
& I\left(\mathbf{Q}^{+\varepsilon}\right)=V^{i-1} \oplus \cdots \oplus V^{1} \oplus \mathbf{Q}^{++} \oplus \mathbf{Q}^{-+} \oplus \mathbf{Q}^{+-} \oplus \mathbf{Q}^{--}
\end{aligned}
$$

Hence,

$$
\phi_{j}^{i}(V)=4\left(\perp_{j<i}\langle-1\rangle_{V_{j}} \perp\langle-1\rangle_{+-} \perp\langle-1\rangle_{++} \perp\langle-1\rangle_{-+} \perp\langle-1\rangle_{--}\right)
$$

in $L_{0}^{h}\left(\mathbf{Q}\left(\zeta_{3}\right) D\left(2^{l}, 2\right)\right)$, and the results of [5] show that the corresponding element in

$$
L_{0}^{h}\left(\mathbf{Z}\left(\zeta_{3}\right) D\left(2^{i}, 2\right)\right)
$$

is not divisible by 2 .

For the quaternion group $Q\left(2^{i}\right)$ we have

LEMMA 6.9. The generators (in 1.28) all have trivial images in $L_{*}^{h}\left(\mathbf{Z}\left(\zeta_{3}\right) Q\left(2^{i}\right) ; \mathbf{Z} / 2\right)$ if their dimension are 4 or greater, while those of dimension $<4$ all have non-trivial (and independent) images.

Proof. The non-triviality of all the generators above is clear for dimensions $\leq 3$. (In dimension 3, this is just the Cappell-Shaneson example as described in [5, $\S 8]$, while in dimensions 1,2 project onto the dihedral group). The triviality of the classes $e_{\zeta_{3}}\left(\left\{M(i), f_{i}\right\}\right)$ follows from an argument completely analogous to that in the proof of 6.8. The point is that the image of $\langle 1\rangle^{+}$for $Z / 2$ is the class $\langle 1\rangle$ in $L_{0}^{h}\left(\mathbf{Q}\left(\zeta_{3}\right) Q\left(2^{i}\right)\right)$, but $\langle 1\rangle \perp\langle T\rangle$ is represented by $2\langle 1\rangle^{+}$. The proof is complete.

Finally, to complete the proof of the results in the introduction we need to consider the extra terms $\left\langle\left(\mathrm{Sq}^{1} V\right)^{2} f^{*}\left(\kappa^{\prime}\right),[M]\right\rangle$ which could possibly appear in the product formulae for the map $e_{\zeta_{3}}$. For oriented $M$ it suffices, as usual to analyze the situation for the model groups, and we have

THEOREM 6.10. For the model groups the coefficients above are identically 0 whenever $M$ is an oriented manifold.

Proof. For $\pi=\mathbf{Z} / 2$ or $\mathbf{Z} / 4$ we know that $L_{3}^{h}\left(\mathbf{Z}\left(\zeta_{3}\right) \pi ; \mathbf{Z} / 2\right)=0$, so $e_{\zeta_{3}}(\tau \circ M(0))=0$ in both these cases. Likewise, when $i>1,6.1$ 
implies $e_{\zeta_{3}}(\tau \circ M(i))=0$. But in any case $e_{\zeta_{3}}(\tau \circ M(i))$ will involve detection by coefficients $\left\langle\left(\mathrm{Sq}^{1} V\right)^{2} f^{*}(b),[M]\right\rangle$, and since $\operatorname{Sq}^{1}(b)=0$, it follows that

$$
\left(\mathrm{Sq}^{1} V\right)^{2} f^{*}(b)=\mathrm{Sq}^{1}\left(V \circ \mathrm{Sq}^{1} V \circ f^{*}(b)\right)
$$

and so will vanish in top dimension on any oriented manifold.

When $\pi=\mathrm{Z} / 2 \times \mathbf{Z} / 2$ we have that $e_{\zeta_{3}}(\tau \circ M(1) \times M(1))$ is represented by either $\tau \circ V$ or 0 from 6.4. But 6.1 implies that $\tau \circ$ $V=0$ so this class vanishes. Otherwise,

$e_{\zeta_{3}}(\tau \circ(M(i) \times M(j)))=e_{\zeta_{3}}((\tau \circ M(i)) \times M(j))=e_{\zeta_{3}}(M(i) \times(\tau \circ M(j))$

and unless both $i, j=1$ the proof for $\mathbf{Z} / 2$ implies that the above class gives 0 in $L_{*}^{h}\left(\mathbf{Z}\left(\zeta_{3}\right) \mathbf{Z} / 2 \times Z / 2 ; Z / 2\right)$.

When $\pi=Q\left(2^{i}\right)$ we dispose of the classes in dimension 1 by noting that they factor through $\mathrm{Z} / 4$, hence $e_{\zeta_{3}}(\tau \circ M(0))$ represents 0 in $L_{3}^{h}\left(\mathbf{Z}\left(\zeta_{3}\right) Q\left(2^{i}\right) ; \mathbf{Z} / 2\right)$. The remaining classes in dimensions 2 and 3 on producting with $\tau$ give formulae involving only the classes in dimensions 2 and 3 in $H^{*}\left(Q\left(2^{i}\right) ; \mathbf{Z} / 2\right)$, but these classes are all in the kernel of $\mathrm{Sq}^{1}$, so 6.11 implies the vanishing of these classes on oriented manifolds and 6.10 follows.

\section{BIBLIOGRAPHY}

[1] I. Hambleton and R. J. Milgram, The surgery obstruction groups for finite 2groups, Invent. Math., 63 (1980) 33-52.

[2] I. Hambleton, R. J. Milgram, L. Taylor, and B. Williams, Surgery with finite fundamental group, Proc. London Math. Soc., (3) 56 (1988), 349-379.

[3] R. J. Milgram, Surgery with coefficients, Ann. of Math., 2 (100) (1974), 194248.

[4] _- The bar construction and Abelian H-spaces, Illinois J. Math., 11 (1967), 242-250.

[5] _- Surgery with finite fundamental group I: the obstructions, Pacific J. Math., 151 (1991), 65-115.

[6] R. J. Milgram and A. Ranicki, Some product formulae for nonsimply connected surgery problems, Trans. Amer. Math. Soc., 297 (1986), 383-413.

[7] _ Witt rings and reciprocity laws for genus 0 function fields, (to appear in Crelle J.)

[8] J. Milnor and J. Stasheff, Characteristic Classes, Ann. Math Studies, vol. 76, Princeton University Press.

[9] J. Morgan and D. Sullivan, The transversality characteristic class and linking cycles in surgery theory, Ann. of Math., 99 (1974), 463-544.

[10] A. Ranicki, The algebraic theory of surgery I, II, Proc. London Math. Soc., 3 (40) (1980), 87-283. 
[11] N. Steenrod and D. Epstein, Cohomology Operations, Ann. Math. Studies, vol. 50, Princeton University Press, 1962.

[12] L. Taylor and B. Williams, Surgery on closed manifolds, mimeo. Notre Dame Univ., 1980.

[13] ___ Surgery spaces, formulae and structure, Proceedings 1978 Waterloo conference on Algebraic Topology, Lecture Notes in Math., vol. \#741, 170-195, Springer-Verlag.

[14] C. T. C. Wall, Formulae for surgery obstructions, Topology, 15 (1976) 189-210.

Received March 23, 1988 and in revised form May 25, 1989. Research supported in part by grants from the NSF.

University of California, SAN Diego

LAJOLla, CA 92093

AND

STANFORD UNIVERSITY

STANFORD, CA 94305-2125 


\section{PACIFIC JOURNAL OF MATHEMATICS EDITORS}

\author{
V. S. VARADARAJAN \\ (Managing Editor) \\ University of California \\ Los Angeles, CA 90024-1555-05 \\ Herbert Clemens \\ University of Utah \\ Salt Lake City, UT 84112 \\ THOMAS ENRIGHT \\ University of California, San Diego \\ La Jolla, CA 92093
}

Nicholas ERCOLANI

University of Arizona

Tucson, AZ 85721

R. FINN

Stanford University

Stanford, CA 94305

VAUGHAN F. R. JONES

University of California

Berkeley, CA 94720

STEVEN KeRCKHOFF

Stanford University

Stanford, CA 94305
C. C. MOORE

University of California

Berkeley, CA 94720

Martin ScharlemanN

University of California

Santa Barbara, CA 93106

HAROLd STARK

University of California, San Diego

La Jolla, CA 92093

\section{ASSOCIATE EDITORS}
R. ARENS
E. F. BECKENBACH
B. H. NeUMANN
F. WolF
K. Yoshida (1906-1982)
(1904-1989)

SUPPORTING INSTITUTIONS

UNIVERSITY OF ARIZONA

UNIVERSITY OF BRITISH COLUMBIA

CALIFORNIA INSTITUTE OF TECHNOLOGY

UNIVERSITY OF CALIFORNIA

MONTANA STATE UNIVERSITY

UNIVERSITY OF NEVADA, RENO

NEW MEXICO STATE UNIVERSITY

OREGON STATE UNIVERSITY
UNIVERSITY OF OREGON

UNIVERSITY OF SOUTHERN CALIFORNIA

STANFORD UNIVERSITY

UNIVERSITY OF HAWAII

UNIVERSITY OF TOKYO

UNIVERSITY OF UTAH

WASHINGTON STATE UNIVERSITY

UNIVERSITY OF WASHINGTON 


\section{Pacific Journal of Mathematics}

\section{Vol. 151, No. $1 \quad$ November, 1991}

Fernanda Maria Botelho, Rotational entropy for annulus endomorphisms . . . 1

David F. Cowan, Some infinite chains in the lattice of varieties of inverse

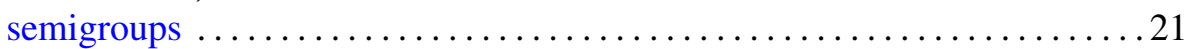

S. Greco and G. Raciti, The Lüroth semigroup of plane algebraic curves . . . 44

Kang-Tae Kim, Automorphism groups of certain domains in $\mathbf{C}^{n}$ with a

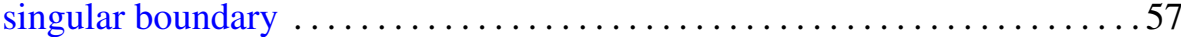

R. James Milgram, Surgery with finite fundamental group. I: The

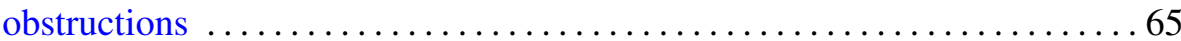

R. James Milgram, Surgery with finite fundamental group. II: The oozing

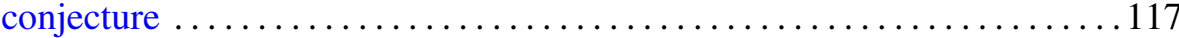

Shahriar Shahriari, On central type factor groups $\ldots \ldots \ldots \ldots \ldots \ldots \ldots 151$

Lynne Walling, Hecke eigenforms and representation numbers of quadratic forms ............................................... 179 